\title{
ON THE FRICTIONLESS UNILATERAL CONTACT OF TWO VISCOELASTIC BODIES
}

\author{
M. BARBOTEU, T.-V. HOARAU-MANTEL, \\ AND M. SOFONEA
}

Received 12 December 2002 and in revised form 10 June 2003

We consider a mathematical model which describes the quasistatic contact between two deformable bodies. The bodies are assumed to have a viscoelastic behavior that we model with Kelvin-Voigt constitutive law. The contact is frictionless and is modeled with the classical Signorini condition with zero-gap function. We derive a variational formulation of the problem and prove the existence of a unique weak solution to the model by using arguments of evolution equations with maximal monotone operators. We also prove that the solution converges to the solution of the corresponding elastic problem, as the viscosity tensors converge to zero. We then consider a fully discrete approximation of the problem, based on the augmented Lagrangian approach, and present numerical results of two-dimensional test problems.

\section{Introduction}

The phenomena of contact between deformable bodies or between deformable and rigid bodies abound in industry and everyday life. A few simple examples are the contact of brake pads with wheels, tires on roads, and pistons with skirts. Common industrial processes, such as metal forming and metal extrusion, involve contact evolutions. Because of the importance of contact process in structural and mechanical systems, considerable effort has been put into modeling, analysis, and numerical simulations. Literature in this field is extensive; books, proceedings, and reviewsdealing with models involving friction, adhesion, or wear of the contact surfaces include $[13,15,24,25,28,30,31$, $34,35]$. For the sake of simplicity and in order to keep this section in a

Copyright @ 2003 Hindawi Publishing Corporation

Journal of Applied Mathematics 2003:11 (2003) 575-603

2000 Mathematics Subject Classification: 74M15, 74S05, 35K85

URL: http:/ /dx.doi.org/10.1155/S1110757X03212043 
reasonable length, we refer in what follows mainly to results and papers concerning frictionless contact problems and, with very few exceptions, we avoid references to frictional models.

The Signorini problem was formulated in [32] as a model of unilateral frictionless contact between an elastic body and a rigid foundation. Mathematical analysis of this problem was first provided in $[11,12]$ and its numerical approximation was described in detail in [24]. Results concerning the frictionless Signorini contact problem between two elastic bodies have been obtained in $[16,17,18,19]$; there, the authors provided existence and uniqueness results of the weak solutions, considered a finite-element model for solving the contact problems, and discussed some solution algorithms.

The first existence result for weak solutions of the quasistatic contact problem with Coulomb's friction and Signorini's condition for an elastic material has been obtained recently in [3]. The proof was based on a sequence of approximations using normal compliance. First, the approximate problems with normal compliance were discretized in time and a priori estimates on their solutions were obtained. Passing to the time discretization limit yielded a solution for the quasistatic problem with normal compliance. Using then a regularity result, based on the shifting technique, the existence to a limit function which solves the quasistatic Signorini frictional problem was obtained. The uniqueness of the solution was left open. Unlike [3], in this paper we deal with frictionless contact between two viscoelastic bodies. We use a different method and establish a unique solution to the model.

In all the references in the previous two paragraphs, it was assumed that the deformable bodies were linearly elastic. However, a number of recent publications is dedicated to the modeling, analysis, and numerical approximation of contact problems involving viscoelastic and viscoplastic materials. Thus, the variational analysis of the frictionless Signorini problem was provided in [33] in the case of rate-type viscoplastic materials and the numerical analysis of this problem was studied in [7]. These results were extended to the frictionless Signorini problem between two viscoplastic bodies in [14,29], respectively. A survey of frictionless contact problems with viscoplastic materials, including numerical experiments for test problems in one, two, and three dimensions, may be found in $[10,15]$. Existence results in the study of the Signorini frictionless contact problem have been obtained in $[20,22]$ in the case of dynamic processes for viscoelastic materials with singular memory and, more recently in [5], in the case of quasistatic process for Kelvin-Voigt materials.

Dynamic frictional contact problems with linearly Kelvin-Voigt viscoelastic materials have been considered in [21, 23]. In [21], the contact 
is modeled with the Signorini condition with zero gap and friction is described with Tresca's law. The existence of a weak solution to the model is obtained in two steps: first, the contact condition is penalized and the solvability of the penalized problems is proved by using the Galerkin approximation; then, compactness and lower semicontinuity arguments are employed to prove that the approximate solutions converge to an element which is shown to be a weak solution to the frictional contact problem. Notice that this result holds, in particular, when the friction bound vanishes, that is, for the Signorini frictionless contact problem; and from this point of view, it represents a dynamic version of the existence and uniqueness result obtained in [5] for the quasistatic model. For the problem studied in [23], the contact is modeled with unilateral conditions in velocities associated to a version of Coulomb's law of dry friction in which the coefficient of friction may depend on the solution. Again, the solvability of the model is proved using penalization and regularization methods. In both papers [21,23], regularity results of the solution are obtained by using a shift technique.

The aim of this paper is to provide variational analysis and numerical simulations in the study of the frictionless contact between two viscoelastic bodies. Since we here consider quasistatic processes for KelvinVoigt viscoelastic materials and the Signorini contact condition, this paper may be considered as a continuation of [5], where the contact between a viscoelastic body and a rigid foundation is investigated. We use arguments similar to those used in [5] in order to prove the wellposedness of the problem, but with a different choice of the spaces and operators since the physical settings, in [5] and here, are different. The other trait of novelty of the present paper consists in the fact that here we obtain an approach to elasticity result, present a fully discrete scheme of the problem, and provide numerical simulations.

The rest of the paper is organized as follows. In Section 2, we state the mechanical problem, list the assumptions on the data, and derive the variational formulation to the model. In Section 3, we provide the existence of a unique weak solution to the mechanical problem. The proof is based on an abstract result on evolution equations with maximal monotone operators and arguments from convex analysis. In Section 4, we investigate the behavior of the solution when the viscosity operator converges to zero. In Section 5, we consider a fully discrete approximation of the problem, based on the finite-difference scheme for the time variable, and the finite-element method for the spatial variable; and in Section 6, we present numerical results in the study of two-dimensional test problems. We conclude the paper in Section 7, where some open problems are described. 


\section{Problem statement and variational formulation}

We consider two viscoelastic bodies which occupy the bounded domains $\Omega^{1}$ and $\Omega^{2}$ of $\mathbb{R}^{d}(d=1,2,3$ in applications). We put a superscript $m$ to indicate that a quantity or subset is related to the domain $\Omega^{m}, m=1,2$. Everywhere in the sequel, $\mathbb{S}^{d}$ represents the space of second-order symmetric tensors on $\mathbb{R}^{d}$, the indices $i, j, k$, and $l$ run between 1 and $d$, and the summation convention over a repeated index is adopted. Moreover, an index that follows a comma indicates a partial derivative with respect to the corresponding component of the spatial variable and a dot above indicates the derivative with respect to the time variable.

For each domain $\Omega^{m}$, the boundary $\Gamma^{m}$ is assumed to be Lipschitz continuous and is partitioned into three disjoint measurable parts $\Gamma_{1}^{m}$, $\Gamma_{2}^{m}$, and $\Gamma_{3}^{m}$, with meas $\Gamma_{1}^{m}>0$. Let $\boldsymbol{v}^{m}=\left(v_{i}^{m}\right)$ be the outward normal to $\Gamma^{m}$. We are interested in the quasistatic process of evolution of the bodies on the time interval $[0, T]$, with $T>0$. The bodies are assumed to be clamped on $\Gamma_{1}^{m} \times(0, T)$ while the volume forces of densities $\boldsymbol{\varphi}_{1}^{m}$ and the surface tractions $\varphi_{2}^{m}$ act on $\Omega^{m} \times(0, T)$ and $\Gamma_{2}^{m} \times(0, T)$, respectively. The two bodies can enter in contact along the common part $\Gamma_{3}^{1}=\Gamma_{3}^{2}=\Gamma_{3}$. The contact is frictionless and is modelled with Signorini condition in a form with a zero-gap function. We assume that the process is quasistatic and we use the Kelvin-Voigt constitutive law to describe the material's behavior. With these assumptions, the mechanical problem we study here may be formulated as follows.

Problem 2.1. For $m=1,2$, find a displacement field $\mathbf{u}^{m}=\left(u_{i}^{m}\right): \Omega^{m} \times[0, T]$ $\rightarrow \mathbb{R}^{d}$ and a stress field $\sigma^{m}=\left(\sigma_{i j}^{m}\right): \Omega^{m} \times[0, T] \rightarrow \mathbb{S}^{d}$ such that

$$
\begin{aligned}
& \boldsymbol{\sigma}^{m}=\mathcal{A}^{m} \boldsymbol{\varepsilon}(\dot{\mathbf{u}})+\mathcal{G}^{m} \boldsymbol{\varepsilon}(\mathbf{u}) \quad \text { in } \Omega^{m} \times(0, T), \\
& \operatorname{Div} \boldsymbol{\sigma}^{m}+\boldsymbol{\varphi}_{1}^{m}=\mathbf{0} \quad \text { in } \Omega^{m} \times(0, T), \\
& \mathbf{u}^{m}=\mathbf{0} \quad \text { on } \Gamma_{1}^{m} \times(0, T), \\
& \boldsymbol{\sigma}^{m} \boldsymbol{v}^{m}=\boldsymbol{\varphi}_{2}^{m} \quad \text { on } \Gamma_{2}^{m} \times(0, T), \\
& u_{v}^{1}+u_{v}^{2} \leq 0, \quad \sigma_{v}^{1}=\sigma_{v}^{2} \leq 0, \quad \text { on } \Gamma_{3} \times(0, T), \\
& \left(u_{v}^{1}+u_{v}^{2}\right) \sigma_{v}^{1}=0, \quad \boldsymbol{\sigma}_{\tau}^{m}=\mathbf{0}, \quad \text { on } \Gamma_{3} \times(0, T), \\
& \mathbf{u}^{m}(0)=\mathbf{u}_{0}^{m} \quad \text { in } \Omega^{m} .
\end{aligned}
$$

Here (2.1) represents the constitutive law in which $\mathscr{A}^{m}$ is a fourthorder tensor, $\mathcal{G}^{m}$ is a nonlinear constitutive function, and

$$
\boldsymbol{\varepsilon}\left(\mathbf{u}^{m}\right)=\left(\varepsilon_{i j}\left(\mathbf{u}^{m}\right)\right)=\left(\frac{1}{2}\left(u_{i, j}^{m}+u_{j, i}^{m}\right)\right)
$$


represents the small strain tensor. Equation (2.2) is the equilibrium equation in which Div $\boldsymbol{\sigma}^{m}=\left(\sigma_{i j, j}^{m}\right)$ denotes the divergence of the tensor-valued function $\sigma^{m}$, and conditions (2.3) and (2.4) are the displacement and traction boundary conditions, respectively. Conditions (2.5) and (2.6) represent the frictionless Signorini conditions in which $u_{v}^{m}, \sigma_{v}^{m}$, and $\boldsymbol{\sigma}_{\tau}^{m}$ are the normal displacement, the normal, and the tangential stress, respectively, given by

$$
\begin{aligned}
& u_{v}^{m}=u_{i}^{m} v_{i}^{m}, \quad \sigma_{i}^{m}=\sigma_{i j}^{m} v_{i}^{m} v_{j}^{m}, \\
& \boldsymbol{\sigma}_{\tau}^{m}=\left(\sigma_{\tau i}^{m}\right)=\left(\sigma_{i j}^{m} v_{j}^{m}-\sigma_{v}^{m} v_{i}^{m}\right) .
\end{aligned}
$$

Finally, (2.7) represents the initial condition in which $\mathbf{u}_{0}^{m}$ is the given initial displacement.

Everywhere in this paper, we denote by "." the inner product on the spaces $\mathbb{R}^{d}$ and $\mathbb{S}^{d}$ and by $|\cdot|$ the Euclidean norms on these spaces. For every element $\mathbf{v} \in H^{1}\left(\Omega^{m}\right)^{d}$, we keep the notation $\mathbf{v}$ for the trace $\gamma \mathbf{v}$ of $\mathbf{v}$ on $\Gamma^{m}$. We introduce the following spaces:

$$
\begin{aligned}
Q^{m} & =\left\{\boldsymbol{\tau}=\left(\tau_{i j}\right) \mid \tau_{i j}=\tau_{j i} \in L^{2}\left(\Omega^{m}\right), 1 \leq i, j \leq d\right\}, \\
H_{1}^{m} & =\left\{\mathbf{u}=\left(u_{i}\right) \mid \boldsymbol{\varepsilon}(\mathbf{u}) \in Q^{m}\right\}, \\
Q_{1}^{m} & =\left\{\boldsymbol{\tau}=\left(\tau_{i j}\right) \mid \operatorname{Div} \boldsymbol{\tau} \in L^{2}\left(\Omega^{m}\right)^{d}\right\}, \\
V^{m} & =\left\{\mathbf{v}=\left(v_{i}\right) \mid v_{i} \in H^{1}\left(\Omega^{m}\right)^{d}, \mathbf{v}=\mathbf{0} \text { on } \Gamma_{1}^{m}, 1 \leq i \leq d\right\} .
\end{aligned}
$$

These are real Hilbert spaces endowed with their canonical inner products denoted by $(\cdot, \cdot)_{X}$ and the associate norms $\|\cdot\|_{X}$, where $X$ is one of these previous spaces. Since meas $\Gamma_{1}^{m}>0$, Korn's inequality holds (see, e.g., [26, page 79]) and therefore

$$
\|\varepsilon(\mathbf{v})\|_{Q^{m}} \geq c_{K}\|\mathbf{v}\|_{H^{1}\left(\Omega^{m}\right)^{d}} \quad \forall \mathbf{v} \in V^{m}, m=1,2
$$

Here $c_{K}$ denotes a positive constant which depends on $\Omega^{m}$ and $\Gamma_{1}^{m}$.

In the study of the mechanical problem (2.1)-(2.7), we make the following assumptions for $m=1,2$. The viscosity tensor $\mathscr{A}^{m}=\left(a_{i j k l}^{m}\right): \Omega^{m} \times$ $\mathbb{S}^{d} \rightarrow \mathbb{S}^{d}$ satisfies the usual properties of symmetry and ellipticity, that is,

$$
\begin{gathered}
a_{i j k l}^{m} \in L^{\infty}\left(\Omega^{m}\right), \\
\mathscr{A}^{m} \boldsymbol{\sigma} \cdot \boldsymbol{\tau}=\boldsymbol{\sigma} \cdot \mathscr{A}^{m} \boldsymbol{\tau} \quad \forall \boldsymbol{\sigma}, \boldsymbol{\tau} \in \mathbb{S}^{d}, \text { a.e. in } \Omega^{m},
\end{gathered}
$$

$\exists c_{\mathscr{A}^{m}}>0$ such that $\mathscr{A}^{m} \boldsymbol{\tau} \cdot \boldsymbol{\tau} \geq c_{\mathscr{A}^{m}}|\boldsymbol{\tau}|^{2} \quad \forall \boldsymbol{\tau} \in \mathbb{S}^{d}$, a.e. in $\Omega^{m}$. 
The elasticity operator $\mathcal{G}^{m}: \Omega^{m} \times \mathbb{S}^{d} \rightarrow \mathbb{S}^{d}$ satisfies the following assumptions:

$$
\begin{array}{r}
\exists L^{m}>0 \text { such that }\left|\mathcal{G}^{m}\left(\mathbf{x}, \boldsymbol{\varepsilon}_{1}\right)-\mathcal{G}^{m}\left(\mathbf{x}, \boldsymbol{\varepsilon}_{2}\right)\right| \leq L^{m}\left|\varepsilon_{1}-\boldsymbol{\varepsilon}_{2}\right| \\
\forall \varepsilon_{1}, \boldsymbol{\varepsilon}_{2} \in \mathbb{S}^{d}, \text { a.e. on } \Omega^{m}, \\
\mathbf{x} \longmapsto \mathcal{G}(\mathbf{x}, \boldsymbol{\varepsilon}) \text { is Lebesgue measurable on } Q^{m} \quad \forall \boldsymbol{\varepsilon} \in \mathbb{S}^{d}, \\
\mathbf{x} \longmapsto \mathcal{G}^{m}(\mathbf{x}, \mathbf{0}) \text { belongs to } Q^{m} .
\end{array}
$$

For the body forces and surface tractions, we assume that

$$
\boldsymbol{\varphi}_{1}^{m} \in W^{1,1}\left(0, T ; L^{2}\left(\Omega^{m}\right)^{d}\right), \quad \boldsymbol{\varphi}_{2}^{m} \in W^{1,1}\left(0, T ; L^{2}\left(\Gamma_{2}^{m}\right)^{d}\right) .
$$

In order to simplify the notations, we define the product spaces

$$
\begin{aligned}
H_{1} & =H_{1}^{1} \times H_{1}^{2}, & V & =V^{1} \times V^{2}, \\
Q & =Q^{1} \times Q^{2}, & Q_{1} & =Q_{1}^{1} \times Q_{1}^{2}
\end{aligned}
$$

and we introduce the notation

$$
\begin{gathered}
\boldsymbol{\varepsilon}(\mathbf{v})=\left(\varepsilon\left(\mathbf{v}^{1}\right), \boldsymbol{\varepsilon}\left(\mathbf{v}^{2}\right)\right) \quad \forall \mathbf{v}=\left(\mathbf{v}^{1}, \mathbf{v}^{2}\right) \in V, \\
\mathcal{A} \boldsymbol{\tau}=\left(\mathcal{A}^{1} \boldsymbol{\tau}^{1}, \mathcal{A}^{2} \boldsymbol{\tau}^{2}\right) \quad \forall \boldsymbol{\tau}=\left(\boldsymbol{\tau}^{1}, \boldsymbol{\tau}^{2}\right) \in Q, \\
\mathcal{G} \boldsymbol{\tau}=\left(\mathcal{G}^{1} \boldsymbol{\tau}^{1}, \mathcal{G}^{2} \boldsymbol{\tau}^{2}\right) \quad \forall \boldsymbol{\tau}=\left(\boldsymbol{\tau}^{1}, \boldsymbol{\tau}^{2}\right) \in Q, \\
\mathbf{u}_{0}=\left(\mathbf{u}_{0}^{1}, \mathbf{u}_{0}^{2}\right) .
\end{gathered}
$$

The spaces $Q$ and $Q_{1}$ are real Hilbert spaces endowed with the canonical inner products denoted by $(\cdot, \cdot)_{Q}$ and $(\cdot, \cdot)_{Q_{1}}$. The associate norms will be denoted by $\|\cdot\|_{Q}$ and $\|\cdot\|_{Q_{1}}$, respectively. Using (2.11) and (2.12), we see that $V$ is a real Hilbert space with the inner product and the associated norm

$$
(\mathbf{u}, \mathbf{v})_{V}=(\mathscr{A} \varepsilon(\mathbf{u}), \varepsilon(\mathbf{v}))_{Q^{\prime}} \quad\|\mathbf{u}\|_{V}=\sqrt{(\mathbf{u}, \mathbf{u})_{V}}, \quad \forall \mathbf{u}, \mathbf{v} \in V
$$

We assume that the initial displacement verifies

$$
\mathbf{u}_{0}=\left(\mathbf{u}_{0}^{1}, \mathbf{u}_{0}^{2}\right) \in U,
$$

where $U$ denotes the set of admissible displacement fields given by

$$
U=\left\{\mathbf{v}=\left(\mathbf{v}^{1}, \mathbf{v}^{2}\right) \in V \mid v_{v}^{1}+v_{v}^{2} \leq 0 \text { on } \Gamma_{3}\right\}
$$


We also define the mapping $\mathbf{f}:[0, T] \rightarrow V$ by

$$
\begin{aligned}
(\mathbf{f}(t), \mathbf{v})_{V}= & \left(\boldsymbol{\varphi}_{1}^{1}(t), \mathbf{v}^{1}\right)_{L^{2}\left(\Omega^{1}\right)^{d}}+\left(\boldsymbol{\varphi}_{1}^{2}(t), \mathbf{v}^{2}\right)_{L^{2}\left(\Omega^{2}\right)^{d}} \\
& +\left(\boldsymbol{\varphi}_{2}^{1}(t), \gamma \mathbf{v}^{2}\right)_{L^{2}\left(\Gamma_{2}^{1}\right)^{d}}+\left(\boldsymbol{\varphi}_{2}^{2}(t), \gamma \mathbf{v}^{2}\right)_{L^{2}\left(\Gamma_{2}^{2}\right)^{d}} \quad \forall \mathbf{v} \in V, t \in[0, T],
\end{aligned}
$$

and we note that conditions (2.14) imply that

$$
\mathbf{f} \in W^{1,1}(0, T ; V)
$$

Using the standard arguments, it can be shown that if the couple of functions $(\mathbf{u}, \boldsymbol{\sigma})$ (where $\mathbf{u}=\left(\mathbf{u}^{1}, \mathbf{u}^{2}\right)$ and $\boldsymbol{\sigma}=\left(\boldsymbol{\sigma}^{1}, \boldsymbol{\sigma}^{2}\right)$ ) is a regular solution of the mechanical Problem 2.1, then

$$
\mathbf{u}(t) \in U, \quad(\boldsymbol{\sigma}(t), \boldsymbol{\varepsilon}(\mathbf{v})-\varepsilon(\mathbf{u}(t)))_{Q} \geq(\mathbf{f}(t), \mathbf{v}-\mathbf{u}(t))_{V} \quad \forall \mathbf{v} \in U, t \in(0, T)
$$

This inequality leads us to consider the following variational problem.

Problem 2.2. Find a displacement field $\mathbf{u}=\left(\mathbf{u}^{1}, \mathbf{u}^{2}\right):[0, T] \rightarrow V$ and a stress field $\sigma=\left(\sigma^{1}, \sigma^{2}\right):[0, T] \rightarrow Q_{1}$ such that

$$
\begin{gathered}
\boldsymbol{\sigma}(t)=\mathcal{A} \varepsilon(\dot{\mathbf{u}}(t))+\mathcal{G} \varepsilon(\mathbf{u}(t)) \quad \text { a.e. } t \in(0, T), \\
\mathbf{u}(t) \in U, \quad(\boldsymbol{\sigma}(t), \boldsymbol{\varepsilon}(\mathbf{v})-\boldsymbol{\varepsilon}(\mathbf{u}(t)))_{Q} \geq(\mathbf{f}(t), \mathbf{v}-\mathbf{u}(t))_{V} \\
\forall \mathbf{v} \in U, \text { a.e. } t \in(0, T), \\
\mathbf{u}(0)=\mathbf{u}_{0} .
\end{gathered}
$$

We remark that Problem 2.2 is formally equivalent to the mechanical problem (2.1)-(2.7). Indeed, if $(\mathbf{u}, \boldsymbol{\sigma})$ represents a regular solution of the variational Problem 2.2, then, using the arguments of [9], it follows that $(\mathbf{u}, \boldsymbol{\sigma})$ satisfies Problem 2.1. For this reason, we may consider Problem 2.2 as the variational formulation of the mechanical problem (2.1)-(2.7).

\section{An existence and uniqueness result}

The main result of this section concerns the existence and uniqueness of the solution of Problem 2.2. The proof is essentially based on the following theorem which is recalled here for the convenience of the reader.

TheOREM 3.1. Let $X$ be a real Hilbert space and let $A: D(A) \subset X \rightarrow 2^{X}$ be a multivalued operator such that the operator $A+\omega I$ is maximal monotone for 
582 Frictionless contact of two viscoelastic bodies

some real $\omega$. Then, for every $f \in W^{1,1}(0, T ; X)$ and $u_{0} \in D(A)$, there exists a unique function $u \in W^{1, \infty}(0, T ; X)$ which satisfies

$$
\begin{gathered}
\dot{u}(t)+A u(t) \ni f(t) \quad \text { a.e. } t \in(0, T), \\
u(0)=u_{0} .
\end{gathered}
$$

Here and below $D(A), 2^{X}$, and $I$ denote, respectively, the domain of the multivalued operator $A$, the set of the subsets of $X$, and the identity map on $X$. The proof of this theorem can be found in [6, page 32].

Now we use Theorem 3.1 to obtain the following existence and uniqueness result.

THEOREM 3.2. Under assumptions (2.12), (2.13), (2.14), and (2.18), there exists a unique solution $(\mathbf{u}, \boldsymbol{\sigma})$ to Problem 2.2, which satisfies

$$
\mathbf{u} \in W^{1, \infty}(0, T ; V), \quad \boldsymbol{\sigma} \in L^{\infty}\left(0, T ; Q_{1}\right) .
$$

Proof. By Riesz representation theorem, we define an operator $\mathbb{B}: V \rightarrow V$ by

$$
(\boldsymbol{B u}, \mathbf{v})_{V}=(\mathcal{G} \varepsilon(\mathbf{u}), \varepsilon(\mathbf{v}))_{Q} \quad \forall \mathbf{u}, \mathbf{v} \in V
$$

From (2.12) and (2.13), we have

$$
\left\|B \mathbf{u}_{1}-\boldsymbol{B} \mathbf{u}_{2}\right\|_{V} \leq \frac{L_{\mathcal{G}}}{m_{A}}\left\|\mathbf{u}_{1}-\mathbf{u}_{2}\right\|_{V} \quad \forall \mathbf{u}_{1}, \mathbf{u}_{2} \in V
$$

where $m_{\mathscr{A}}=\inf \left(c_{A^{1}}, c_{A^{2}}\right)$, which proves that $B$ is a Lipschitz continuous operator. So, the operator $\boldsymbol{B}+\left(L_{\mathcal{G}} / m_{\mathcal{A}}\right) I: V \rightarrow V$ is a monotone Lipschitz continuous operator. We now introduce the indicator function $\psi u$ of the set $U$ and its subdifferential $\partial \psi u: V \rightarrow 2^{V}$. Since the set $U$ is a nonempty, closed, and convex part of the space, the subdifferential $\partial \psi_{U}$ is a maximal monotone operator on $V$ and, moreover, $D(\partial \psi U)=U$.

We can now say that the sum $\partial \psi U+B+\left(L_{\mathcal{G}} / m_{A}\right) I: U \subseteq V \rightarrow 2^{V}$ is a maximal monotone operator. Keeping in mind assumptions (2.21) and (2.18), we can apply Theorem 3.1 with $X=V, A=\partial \psi u+B$, and $\omega=$ $L_{\mathcal{G}} / m_{\AA}$. We deduce that there exists a unique element $\mathbf{u} \in W^{1, \infty}(0, T ; V)$ such that

$$
\begin{gathered}
\dot{\mathbf{u}}(t)+\partial \psi U(\mathbf{u}(t))+B \mathbf{u}(t) \ni \mathbf{f}(t) \quad \text { a.e. } t \in(0, T), \\
\mathbf{u}(0)=\mathbf{u}_{0} .
\end{gathered}
$$


Form (3.3), (3.5), and (2.17), we obtain

$$
\begin{gathered}
\mathbf{u}(t) \in U, \\
(\mathcal{A} \varepsilon(\dot{\mathbf{u}}(t)), \varepsilon(\mathbf{v})-\varepsilon(\mathbf{u}(t)))_{Q}+(\mathcal{G} \varepsilon(\mathbf{u}(t)), \varepsilon(\mathbf{v})-\varepsilon(\mathbf{u}(t)))_{Q} \\
\geq(\mathbf{f}(t), \mathbf{v}-\mathbf{u}(t))_{V} \quad \forall \mathbf{v} \in U \text {, a.e. } t \in(0, T) .
\end{gathered}
$$

Now let $\sigma$ denote the function defined by (2.23). From (3.7) and (3.6), it follows that the couple of functions $(\mathbf{u}, \boldsymbol{\sigma})$ solves Problem 2.2. Moreover, from the regularity $\mathbf{u} \in W^{1, \infty}(0, T ; V)$ and assumptions (2.12) and (2.13), we obtain $\sigma \in L^{\infty}(0, T ; Q)$. It now follows from (2.24) and (2.20) that

$$
\operatorname{Div} \boldsymbol{\sigma}^{m}+\boldsymbol{\varphi}_{1}^{m}=0 \quad \text { in } \Omega^{m} \times(0, T),
$$

and, keeping in mind (2.14), we obtain $\sigma \in L^{\infty}\left(0, T ; Q_{1}\right)$, which concludes the existence part of the proof.

The uniqueness part results from the uniqueness of the element $\mathbf{u} \in$ $W^{1, \infty}(0, T ; V)$ which solves (3.5) and (3.6), guaranteed by Theorem 3.1.

We conclude by Theorem 3.2 that, under assumptions (2.12), (2.13), (2.14), and (2.18), the mechanical problem (2.1)-(2.7) has a unique weak solution, which solves Problem 2.2.

\section{Approach to elasticity}

In this section, we investigate the behavior of the solution to Problem 2.2 when the coefficient of viscosity converges to zero. To this end, we restrict ourselves to the linear case. Thus, the function $\mathcal{G}^{m}=\left(g_{i j k l}^{m}\right): \Omega^{m} \times$ $\mathbb{S}^{d} \rightarrow \mathbb{S}^{d}$ will represent below a fourth-order tensor field which satisfies the following assumptions, for $m=1,2$ :

$$
\begin{gathered}
g_{i j k l}^{m} \in L^{\infty}\left(\Omega^{m}\right), \\
\mathcal{G}^{m} \boldsymbol{\sigma} \cdot \boldsymbol{\tau}=\boldsymbol{\sigma} \cdot \mathcal{G}^{m} \boldsymbol{\tau} \quad \forall \boldsymbol{\sigma}, \boldsymbol{\tau} \in \mathbb{S}^{d}, \text { a.e. in } \Omega^{m}, \\
\exists \mathcal{C}_{\mathcal{G}^{m}}>0 \text { such that } \mathcal{G}^{m} \boldsymbol{\tau} \cdot \boldsymbol{\tau} \geq \mathcal{C}_{\mathcal{G}^{m}}|\boldsymbol{\tau}|^{2} \quad \forall \boldsymbol{\tau} \in \mathbb{S}^{d} \text {, a.e. in } \Omega^{m} .
\end{gathered}
$$

Let $\theta>0$. We replace in (2.23) the viscosity operators $\mathscr{A}^{m}$ by $\theta \mathscr{A}^{m}$ and use in what follows the notation $\theta \mathscr{A}=\left(\theta \mathscr{A}^{1}, \theta \mathscr{A}^{2}\right)$. We assume everywhere in this section that, (2.12), (2.14), (2.18), and (4.1) hold and we consider the following variational problem. 
584 Frictionless contact of two viscoelastic bodies

Problem 4.1. Find a displacement field $\mathbf{u}_{\theta}=\left(\mathbf{u}_{\theta}^{1}, \mathbf{u}_{\theta}^{2}\right):[0, T] \rightarrow V$ and a stress field $\sigma_{\theta}=\left(\sigma_{\theta}^{1}, \sigma_{\theta}^{2}\right):[0, T] \rightarrow Q_{1}$ such that

$$
\begin{gathered}
\boldsymbol{\sigma}_{\theta}(t)=\theta \mathcal{A} \varepsilon\left(\dot{\mathbf{u}}_{\theta}(t)\right)+\mathcal{G} \varepsilon\left(\mathbf{u}_{\theta}(t)\right) \quad \text { a.e. } t \in(0, T), \\
\mathbf{u}_{\theta}(t) \in U, \quad\left(\boldsymbol{\sigma}_{\theta}(t), \varepsilon(\mathbf{v})-\varepsilon\left(\mathbf{u}_{\theta}(t)\right)\right)_{Q} \geq\left(\mathbf{f}(t), \mathbf{v}-\mathbf{u}_{\theta}(t)\right)_{V} \\
\forall \mathbf{v} \in U \text {, a.e. } t \in(0, T), \\
\mathbf{u}_{\theta}(0)=\mathbf{u}_{0} .
\end{gathered}
$$

Using Theorem 3.2, it follows that the variational Problem 4.1 has a unique solution $\left(\mathbf{u}_{\theta}, \boldsymbol{\sigma}_{\theta}\right)$ with regularity $\mathbf{u}_{\theta} \in W^{1, \infty}(0, T ; V)$ and $\boldsymbol{\sigma}_{\theta} \in$ $L^{\infty}\left(0, T ; Q_{1}\right)$.

We now introduce the following variational problem.

Problem 4.2. Find a displacement field $\mathbf{u}=\left(\mathbf{u}^{1}, \mathbf{u}^{2}\right):[0, T] \rightarrow V$ and a stress field $\boldsymbol{\sigma}=\left(\boldsymbol{\sigma}^{1}, \boldsymbol{\sigma}^{2}\right):[0, T] \rightarrow Q_{1}$ such that, for all $t \in[0, T]$,

$$
\begin{gathered}
\boldsymbol{\sigma}(t)=\mathcal{G}_{\mathcal{G}}(\mathbf{u}(t)), \\
\mathbf{u}(t) \in U, \quad(\boldsymbol{\sigma}(t), \boldsymbol{\varepsilon}(\mathbf{v})-\boldsymbol{\varepsilon}(\mathbf{u}(t)))_{Q} \geq(\mathbf{f}(t), \mathbf{v}-\mathbf{u}(t))_{V} \quad \forall \mathbf{v} \in U .
\end{gathered}
$$

Clearly Problem 4.2 represents the variational formulation of the Signorini frictionless contact problem between two deformable bodies when the viscoelastic constitutive law (4.2) is replaced by the elastic constitutive law (4.5). Keeping in mind assumptions (2.12), (2.13), (2.14), (2.18), (4.1) and using arguments on elliptic variational inequalities, we deduce that the variational Problem 4.2 has a unique solution $(\mathbf{u}, \boldsymbol{\sigma})$ which has the regularity $\mathbf{u} \in W^{1,1}(0, T ; V)$ and $\boldsymbol{\sigma} \in W^{1,1}\left(0, T ; Q_{1}\right)$.

We consider the following additional assumptions:

$$
\begin{gathered}
\mathbf{u}(0)=\mathbf{u}_{0}, \\
\mathbf{f} \in W^{1,2}(0, T ; V), \quad \mathbf{u}_{\theta} \in W^{2,2}(0, T ; V) .
\end{gathered}
$$

Our main result in this section is the following.

Theorem 4.3. Assume that (2.12), (2.14), (2.18), and (4.1) hold. Then

$$
\mathbf{u}_{\theta} \longrightarrow \mathbf{u} \text { in } L^{2}(0, T ; V) \text { as } \theta \longrightarrow 0
$$


Moreover, if (4.7) holds, then

$$
\max _{s \in[0, T]}\left\|\mathbf{u}_{\theta}(s)-\mathbf{u}(s)\right\|_{V} \longrightarrow 0 \quad \text { as } \theta \longrightarrow 0
$$

and, if (4.8) holds, then

$$
\boldsymbol{\sigma}_{\theta} \longrightarrow \boldsymbol{\sigma} \text { in } L^{2}\left(0, T ; Q_{1}\right) \text { as } \theta \longrightarrow 0
$$

We conclude by these results that the weak solution of the Signorini frictionless contact problem between two elastic bodies may be approached by the weak solution of the Signorini frictionless contact problem between two viscoelastic bodies, as the coefficient of viscosity is small enough. Notice that the convergence (4.9) holds under the basic regularity of the solution, the convergence (4.10) holds under a compatibility condition between the initial and boundary data, and, finally, the convergence (4.11) holds under additional regularity of the data and the solution. In addition to the mathematical interest in the convergences (4.9), (4.10), and (4.11), they are of importance from the mechanical point of view, as they indicate that the frictionless elasticity may be considered as a limit case of frictionless viscoelasticity.

Proof. Let $\theta>0$. We substitute (4.2) into (4.3) and (4.5) into (4.6), respectively, to obtain

$$
\begin{gathered}
\theta\left(\mathcal{A} \varepsilon\left(\dot{\mathbf{u}}_{\theta}(s)\right)+\mathcal{G} \varepsilon\left(\mathbf{u}_{\theta}(s)\right), \boldsymbol{\varepsilon}(\mathbf{v})-\boldsymbol{\varepsilon}\left(\mathbf{u}_{\theta}(s)\right)\right)_{Q} \geq\left(\mathbf{f}(s), \mathbf{v}-\mathbf{u}_{\theta}(s)\right)_{V^{\prime}} \\
(\mathcal{G} \varepsilon(\mathbf{u}(s)), \boldsymbol{\varepsilon}(\mathbf{v})-\boldsymbol{\varepsilon}(\mathbf{u}(s)))_{Q} \geq(\mathbf{f}(s), \mathbf{v}-\mathbf{u}(s))_{V^{\prime}}
\end{gathered}
$$

for all $\mathbf{v} \in U$, a.e. $s \in(0, T)$. Taking $\mathbf{v}=\mathbf{u}(s)$ and $\mathbf{v}=\mathbf{u}_{\theta}(s)$ in the first and the second inequalities, respectively, and adding the resulted relations, we deduce that

$$
\begin{aligned}
\theta\left(\mathcal{A} \varepsilon\left(\dot{\mathbf{u}}_{\theta}(s)\right)-\mathcal{A} \boldsymbol{\varepsilon}(\dot{\mathbf{u}}(s)), \boldsymbol{\varepsilon}\left(\mathbf{u}_{\theta}(s)\right)-\boldsymbol{\varepsilon}(\mathbf{u}(s))\right)_{Q} \\
\quad+\left(\mathcal{G} \varepsilon\left(\mathbf{u}_{\theta}(s)\right)-\mathcal{G} \varepsilon(\mathbf{u}(s)), \boldsymbol{\varepsilon}\left(\mathbf{u}_{\theta}(s)\right)-\boldsymbol{\varepsilon}(\mathbf{u}(s))\right)_{Q} \\
\leq \theta\left(\mathcal{A} \varepsilon(\dot{\mathbf{u}}(s)), \boldsymbol{\varepsilon}(\mathbf{u}(s))-\boldsymbol{\varepsilon}\left(\mathbf{u}_{\theta}(s)\right)\right)_{Q} \quad \text { a.e. } s \in(0, T) .
\end{aligned}
$$

Using (2.17) and the inequality

$$
a b \leq \frac{\theta}{2 \alpha} a^{2}+\frac{\alpha}{2 \theta} b^{2} \quad \forall a, b, \alpha>0
$$


586 Frictionless contact of two viscoelastic bodies

we find that

$$
\begin{aligned}
\theta\left(\dot{\mathbf{u}}_{\theta}(s)\right. & \left.-\dot{\mathbf{u}}(s), \mathbf{u}_{\theta}(s)-\mathbf{u}(s)\right)_{V} \\
& +\left(\mathcal{G} \varepsilon\left(\mathbf{u}_{\theta}(s)\right)-\mathcal{G} \varepsilon(\mathbf{u}(s)), \varepsilon\left(\mathbf{u}_{\theta}(s)\right)-\varepsilon(\mathbf{u}(s))\right)_{Q} \\
\leq & \theta\left(\frac{\theta}{2 m_{\mathcal{G}}}\|\mathcal{A} \varepsilon(\dot{\mathbf{u}}(s))\|_{Q}^{2}+\frac{m_{\mathcal{G}}}{2 \theta}\left\|\varepsilon\left(\mathbf{u}_{\theta}(s)\right)-\varepsilon(\mathbf{u}(s))\right\|_{Q}^{2}\right)
\end{aligned}
$$

a.e. on $(0, T)$,

where $m_{\mathcal{G}_{1}}=\inf \left(c_{\mathcal{G}^{1}}, c_{\mathcal{G}^{2}}\right)$.

Let $t \in[0, T]$. Integrating the previous inequality on $[0, t]$ and using (2.12), (4.1), and (4.4), it follows that

$$
\begin{gathered}
\theta\left\|\mathbf{u}_{\theta}(t)-\mathbf{u}(t)\right\|_{V}^{2}+C_{1} \int_{0}^{t}\left\|\mathbf{u}_{\theta}(s)-\mathbf{u}(s)\right\|_{V}^{2} d s \\
\leq C_{2} \theta^{2} \int_{0}^{t}\|\dot{\mathbf{u}}(s)\|_{V}^{2} d s+C_{3} \theta\left\|\mathbf{u}_{0}-\mathbf{u}(0)\right\|_{V^{\prime}}^{2}
\end{gathered}
$$

which implies that

$$
\left\|\mathbf{u}_{\theta}(t)-\mathbf{u}(t)\right\|_{V}^{2} \leq C_{2} \theta \int_{0}^{t}\|\dot{\mathbf{u}}(s)\|_{V}^{2} d s+C_{3}\left\|\mathbf{u}_{0}-\mathbf{u}(0)\right\|_{V}^{2} .
$$

Here and below, $C_{p}(p=1,2, \ldots)$ represent positive constants which may depend on the problem data but do not depend on time nor on $\theta$.

The convergence result (4.9) now follows from (4.16). Moreover, if (4.7) holds, the convergence (4.10) follows from (4.17).

Assume in the sequel that (4.8) holds; in this case $\sigma_{\theta} \in W^{1,2}(0, T ; Q)$ and, moreover (4.2) and (4.3) hold for all $t \in[0, T]$. Using (4.2), (4.5), (2.12), (4.1), and (2.17), we have

$$
\left\|\boldsymbol{\sigma}_{\theta}(s)-\boldsymbol{\sigma}(s)\right\|_{Q} \leq C_{4}\left(\theta\left\|\dot{\mathbf{u}}_{\theta}(s)\right\|_{V}+\left\|\mathbf{u}_{\theta}(s)-\mathbf{u}(s)\right\|_{V}\right) \quad \forall s \in[0, T],
$$

which implies that

$$
\left\|\boldsymbol{\sigma}_{\theta}-\boldsymbol{\sigma}\right\|_{L^{2}(0, T ; Q)}^{2} \leq C_{5}\left(\theta^{2} \int_{0}^{T}\left\|\dot{\mathbf{u}}_{\theta}(s)\right\|_{V}^{2} d s+\int_{0}^{T}\left\|\mathbf{u}_{\theta}(s)-\mathbf{u}(s)\right\|_{V}^{2} d s\right)
$$

From (4.3), it follows that

$$
\begin{aligned}
\left(\boldsymbol{\sigma}_{\theta}(s+h)-\boldsymbol{\sigma}_{\theta}(s),\right. & \left.\boldsymbol{\varepsilon}\left(\mathbf{u}_{\theta}(s+h)\right)-\boldsymbol{\varepsilon}\left(\mathbf{u}_{\theta}(s)\right)\right)_{Q} \\
\leq & \left(\mathbf{f}(s+h)-\mathbf{f}(s), \mathbf{u}_{\theta}(s+h)-\mathbf{u}_{\theta}(s)\right)_{V^{\prime}}
\end{aligned}
$$


for all $s, h$ such that $s, s+h \in[0, T]$. We deduce from the previous inequality that

$$
\left(\dot{\boldsymbol{\sigma}}_{\theta}(s), \boldsymbol{\varepsilon}\left(\dot{\mathbf{u}}_{\theta}(s)\right)\right)_{Q} \leq\left(\dot{\mathbf{f}}(s), \dot{\mathbf{u}}_{\theta}(s)\right)_{V} \quad \text { a.e. } s \in(0, T) .
$$

Keeping in mind the regularity $\boldsymbol{\sigma}_{\theta} \in W^{1,2}(0, T ; Q)$, we derive (4.2) with respect to the time and plug the result in the previous inequality to obtain

$$
\begin{array}{r}
\theta\left(\mathcal{A} \varepsilon\left(\ddot{\mathbf{u}}_{\theta}(s)\right), \varepsilon\left(\dot{\mathbf{u}}_{\theta}(s)\right)\right)_{Q}+\left(\mathcal{G} \varepsilon\left(\dot{\mathbf{u}}_{\theta}(s)\right), \varepsilon\left(\dot{\mathbf{u}}_{\theta}(s)\right)\right)_{Q} \\
\leq\left(\dot{\mathbf{f}}(s), \dot{\mathbf{u}}_{\theta}(s)\right)_{V} \text { a.e. } s \in(0, T) .
\end{array}
$$

Let again $t$ be fixed on $[0, T]$. Integrating the previous inequality on $[0, t]$ and keeping in mind (4.1) and (4.14), we have

$$
\left\|\dot{\mathbf{u}}_{\theta}(t)\right\|_{V}^{2}+\frac{C_{6}}{\theta} \int_{0}^{t}\left\|\dot{\mathbf{u}}_{\theta}(s)\right\|_{V}^{2} d s \leq C_{7}\left(\frac{1}{\theta}+\left\|\dot{\mathbf{u}}_{\theta}(0)\right\|_{V}^{2}\right),
$$

where $C_{7}$ depends on $\|\dot{\mathbf{f}}\|_{L^{2}(0, T ; V)}$. We multiply this inequality by $e^{\left(C_{6} / \theta\right) t}$ and integrate the result on $[0, T]$ to obtain

$$
\int_{0}^{T} \frac{d}{d t}\left(e^{\left(C_{6} / \theta\right) t} \int_{0}^{t}\left\|\dot{\mathbf{u}}_{\theta}(s)\right\|_{V}^{2} d s\right) d t \leq C_{7}\left(\frac{1}{\theta}+\left\|\dot{\mathbf{u}}_{\theta}(0)\right\|_{V}^{2}\right) \int_{0}^{T} e^{\left(C_{6} / \theta\right) t} d t,
$$

which implies that

$$
e^{\left(C_{6} / \theta\right) T} \int_{0}^{T}\left\|\dot{\mathbf{u}}_{\theta}(s)\right\|_{V}^{2} d s \leq \frac{C_{7}}{C_{6}}\left(1+\theta\left\|\dot{\mathbf{u}}_{\theta}(0)\right\|_{V}^{2}\right)\left(e^{\left(C_{6} / \theta\right) T}-1\right) .
$$

We conclude that

$$
\int_{0}^{T}\left\|\dot{\mathbf{u}}_{\theta}(t)\right\|_{V}^{2} d t \leq C_{8}\left(1+\theta\left\|\dot{\mathbf{u}}_{\theta}(0)\right\|_{V}^{2}\right)
$$

where $C_{8}=C_{7} / C_{6}$.

Let $h>0$ be such that $t+h, t-h \in[0, T]$. We take successively $\mathbf{v}=$ $\mathbf{u}_{\theta}(t+h)$ and $\mathbf{v}=\mathbf{u}_{\theta}(t-h)$ in (4.3) and pass to the limit as $h \rightarrow 0$ in the corresponding inequalities to obtain

$$
\left(\sigma_{\theta}(t), \varepsilon\left(\dot{\mathbf{u}}_{\theta}(t)\right)\right)_{Q}=\left(\mathbf{f}(t), \dot{\mathbf{u}}_{\theta}(t)\right)_{V} .
$$


Next, passing to the limit as $t \rightarrow 0$ in the previous equality and using the regularity $\mathbf{u}_{\theta} \in W^{1,2}(0, T ; V)$ yield

$$
\left(\boldsymbol{\sigma}_{\theta}(0), \boldsymbol{\varepsilon}\left(\dot{\mathbf{u}}_{\theta}(0)\right)\right)_{Q}=\left(\mathbf{f}(0), \dot{\mathbf{u}}_{\theta}(0)\right)_{V}
$$

Now, we write (4.2) at $t=0$, plug the result on the previous equality, and use (2.12), (2.17) and (4.4) to find that

$$
\theta\left\|\dot{\mathbf{u}}_{\theta}(0)\right\|_{V} \leq C_{9}
$$

We multiply (4.26) by $\theta^{2}$ and use (4.29) to obtain

$$
\theta^{2} \int_{0}^{T}\left\|\dot{\mathbf{u}}_{\theta}(t)\right\|_{V}^{2} d t \leq C_{8} \theta^{2}+C_{8} C_{9} \theta
$$

Keeping in mind (3.8), we have

$$
\operatorname{Div} \boldsymbol{\sigma}_{\theta}^{m}+\boldsymbol{\varphi}_{1}^{m}=0 \quad \text { in } \Omega^{m} \times(0, T),
$$

and, using (4.6), we deduce that

$$
\operatorname{Div} \boldsymbol{\sigma}^{m}+\boldsymbol{\varphi}_{1}^{m}=0 \quad \text { in } \Omega^{m} \times(0, T),
$$

for $m=1,2$. Therefore, we obtain

$$
\left\|\sigma_{\theta}(t)-\sigma(t)\right\|_{Q_{1}}=\left\|\sigma_{\theta}(t)-\sigma(t)\right\|_{Q} \quad \forall t \in[0, T]
$$

and, using (4.19) and (4.30), we find that

$$
\left\|\boldsymbol{\sigma}_{\theta}-\boldsymbol{\sigma}\right\|_{L^{2}\left(0, T ; Q_{1}\right)}^{2} \leq C_{5}\left(C_{8} \theta^{2}+C_{8} C_{9} \theta+\int_{0}^{T}\left\|\mathbf{u}_{\theta}(t)-\mathbf{u}(t)\right\|_{V}^{2} d t\right) .
$$

The convergence result (4.11) is now a consequence of (4.9) and (4.34).

\section{Numerical solution}

In this section, we introduce our numerical algorithm, which is based on the Euler-Newton method. To this end, we use a hybrid formulation of the contact problem, based on the augmented Lagrangian approach.

We start with a fully discrete approach of the problem. Let $V^{h}$ be a finite-element subspace of $V$ and define the discrete set of admissible displacements, $U^{h}=U \cap V^{h}$. We denote by $D^{h}: V \rightarrow U^{h}$ the projection operator and, in addition to the finite-dimensional discretization, we 
consider a time partition $\bigcup_{n=1}^{N}\left[t_{n-1}, t_{n}\right]$ of the interval $[0, T]$ such that $0=$ $t_{0}<t_{1}<\cdots<t_{N}=T$. Here, for simplicity, we take $t_{n}=n / N, n=0, \ldots, N$, that is, the partition is equidistant. We note the pointwise values $\mathbf{u}\left(t_{n}\right)$ by $\mathbf{u}_{n}^{h}$ and we recursively define the incremental velocity by the formula

$$
\begin{aligned}
\mathbf{v}_{n}^{h} & =\frac{\mathbf{u}_{n}^{h}-\mathbf{u}_{n-1}^{h}}{\alpha \Delta t}-\frac{(1-\alpha)}{\alpha} \mathbf{v}_{n-1}^{h} \quad \text { if } \alpha \neq 0, \\
\mathbf{v}_{n-1}^{h} & =\frac{\mathbf{u}_{n}^{h}-\mathbf{u}_{n-1}^{h}}{\Delta t} \quad \text { if } \alpha=0,
\end{aligned}
$$

for $n=1,2, \ldots, N$. Here $\Delta t=T /(N+1)$ and $\alpha$ is a parameter introduced in order to adjust the finite-differences scheme. The discretization method based on formula (5.1) is called " $\alpha$-method." Notice that for $\alpha=0$ or 1 , the method is the well-known explicit or implicit Euler method, respectively. Moreover, while $\alpha=1 / 2$, the method is the trapezes method. In order to eliminate instabilities, in what follows we restrict ourselves to the case $\alpha \neq 0$.

Under these considerations and taking into account (2.23), (2.24), and (2.25), a fully discrete approximation of Problem 2.2 is presented as follows.

Problem 5.1. Find $\left\{\mathbf{u}_{n}^{h}\right\}_{n=0, \ldots, N} \subset U^{h}$ such that $\mathbf{u}_{0}^{h}=p^{h} \mathbf{u}_{0}$ and, for $n=1$, $2, \ldots, N$,

$$
\begin{aligned}
\left(\mathcal{A} \varepsilon\left(\mathbf{v}_{n}^{h}\right), \boldsymbol{\varepsilon}\left(\mathbf{w}^{h}\right)-\boldsymbol{\varepsilon}\left(\mathbf{u}_{n}^{h}\right)\right)_{Q}+ & \left(\mathcal{G} \varepsilon\left(\mathbf{u}_{n}^{h}\right), \boldsymbol{\varepsilon}\left(\mathbf{w}^{h}\right)-\boldsymbol{\varepsilon}\left(\mathbf{u}_{n}^{h}\right)\right)_{Q} \\
& \geq\left(\mathbf{f}\left(t_{n}\right), \mathbf{w}^{h}-\mathbf{u}_{n}^{h}\right)_{V} \quad \forall \mathbf{w}^{h} \in U^{h} .
\end{aligned}
$$

To present the solution algorithm, we assume in the sequel that the viscosity and the elasticity operators $\mathscr{A}^{m}: \Omega^{m} \times \mathbb{S}^{d} \rightarrow \mathbb{S}^{d}$ and $\mathcal{G}^{m}: \Omega^{m} \times$ $\mathbb{S}^{d} \rightarrow \mathbb{S}^{d}$ are linear, symmetric, and positively defined, that is, they satisfy conditions (2.12) and (4.1), respectively. We need these assumptions in order to obtain the equivalence between Problem 5.1 and a minimization problem. Moreover, for a virtual displacement field $\mathbf{w}^{h} \in V^{h}$, we use in the sequel the notation $\boldsymbol{\theta}_{n}^{h}\left(\mathbf{w}^{h}\right) \in V^{h}$ for the incremental virtual velocity defined by

$$
\boldsymbol{\theta}_{n}^{h}\left(\mathbf{w}^{h}\right)=\frac{\mathbf{w}^{h}-\mathbf{u}_{n-1}^{h}}{\alpha \Delta t}-\frac{(1-\alpha)}{\alpha} \mathbf{v}_{n-1}^{h} \quad \text { for } n=1,2, \ldots, N
$$

Notice that from (5.1) and (5.3), it follows that $\boldsymbol{\theta}_{n}^{h}\left(\mathbf{u}_{n}^{h}\right)=\mathbf{v}_{n}^{h}$, for $n=1$, $2, \ldots, N$. 
For $n=1,2, \ldots, N$, we define the energy function $\Phi_{n}^{h}: V^{h} \rightarrow \mathbb{R}$ by

$$
\begin{aligned}
\Phi_{n}^{h}\left(\mathbf{w}^{h}\right)= & \frac{1}{2} \int_{\Omega} \mathcal{A} \varepsilon\left(\boldsymbol{\theta}_{n}^{h}\left(\mathbf{w}^{h}\right)\right) \cdot \boldsymbol{\varepsilon}\left(\mathbf{w}^{h}\right) d x \\
& +\frac{1}{2} \int_{\Omega} \mathcal{G} \varepsilon\left(\mathbf{w}^{h}\right) \cdot \boldsymbol{\varepsilon}\left(\mathbf{w}^{h}\right) d x-\left(\mathbf{f}\left(t_{n}\right), \mathbf{w}^{h}\right)_{V} \quad \forall \mathbf{w}^{h} \in V^{h} .
\end{aligned}
$$

Keeping in mind this notation, it is straightforward to see that Problem 5.1 is equivalent to the following problem.

Problem 5.2. Find $\left\{\mathbf{u}_{n}^{h}\right\}_{n=0, \ldots, N} \subset U^{h}$ such that $\mathbf{u}_{0}^{h}=p^{h} \mathbf{u}_{0}$ and, for $n=1$, $2, \ldots, N$,

$$
\Phi_{n}^{h}\left(\mathbf{u}_{n}^{h}\right) \leq \Phi_{n}^{h}\left(\mathbf{w}^{h}\right) \quad \forall \mathbf{w}^{h} \in U^{h}
$$

In order to relax the contact boundary condition on $\Gamma_{3}$, we introduce the indicator function $\left.\left.\psi_{\mathbb{R}^{+}}: \mathbb{R} \rightarrow\right]-\infty,+\infty\right]$ of the set $\mathbb{R}^{+}$and we denote

$$
\mathcal{K}^{h}\left(\mathbf{w}^{h}\right)=\int_{\Gamma_{3}} \psi_{\mathbb{R}^{+}}\left(d_{v}^{h}\left(\mathbf{w}^{h}\right)\right) d a \quad \forall \mathbf{w}^{h} \in V^{h} .
$$

Here $d_{v}^{h}$ represents the positive normal distance defined by $d_{v}^{h}\left(\mathbf{w}^{h}\right)=$ $-\left(w_{v}^{h 1}+w_{v}^{h 2}\right)$ for all $\mathbf{w}^{h} \in V^{h}$. We can now restate Problem 5.2 to obtain the following problem without constraints.

Problem 5.3. Find $\left\{\mathbf{u}_{n}^{h}\right\}_{n=0, \ldots, N} \subset V^{h}$ such that $\mathbf{u}_{0}^{h}=p^{h} \mathbf{u}_{0}$ and, for $n=1$, $2, \ldots, N$,

$$
\Phi_{n}^{h}\left(\mathbf{u}_{n}^{h}\right)+\mathcal{K}^{h}\left(\mathbf{u}_{n}^{h}\right) \leq \Phi_{n}^{h}\left(\mathbf{w}^{h}\right)+\mathcal{K}^{h}\left(\mathbf{w}^{h}\right) \quad \forall \mathbf{w}^{h} \in V^{h}
$$

We now use an augmented Lagrangian approach. To this end, additional immaterial nodes for the Lagrange multipliers have to be considered. The construction of these nodes depends on the contact element we use for the geometrical discretisation of the interface $\Gamma_{3}$. We define

$$
H_{c}^{h}=\left\{\gamma^{h}: \Gamma_{3} \longrightarrow \mathbb{R},\left.\gamma^{h}\right|_{\mathrm{CE}_{s}^{h}}=\text { constant } \forall s=1, \ldots, N_{3}^{c}\right\},
$$

where $N_{3}^{c}$ represents the number of contact elements of the family $\left(\mathrm{CE}_{s}^{h}\right)_{s}$. Notice that $H_{c}^{h}$ is a finite-dimensional subspace of the space $L^{2}\left(\Gamma_{3}\right)$ and will be endowed with its canonical inner product denoted by $(\cdot, \cdot)_{H_{c}^{h}}$. A smooth minimisation problem equivalent to Problem 5.3 is the following. 
Problem 5.4. Find $\left\{\mathbf{u}_{n}^{h}\right\}_{n=0, \ldots, N} \subset V^{h}$ and $\left\{\lambda_{n}^{h}\right\}_{n=0, \ldots, N} \subset H_{c}^{h}$ such that $\mathbf{u}_{0}^{h}=$ $p^{h} \mathbf{u}_{0}$ and, for $n=1,2, \ldots, N$,

$$
\Phi_{n}^{h}\left(\mathbf{u}_{n}^{h}\right)+\mathcal{L}^{h}\left(\mathbf{u}_{n}^{h}, \lambda_{n}^{h}\right) \leq \Phi_{n}^{h}\left(\mathbf{w}^{h}\right)+\mathcal{L}^{h}\left(\mathbf{w}^{h}, \gamma^{h}\right) \quad \forall \mathbf{w}^{h} \in V^{h}, \gamma^{h} \in H_{c}^{h}
$$

Here $\mathcal{L}^{h}\left(\mathbf{u}_{n}^{h}, \lambda_{n}^{h}\right), \lambda_{n}^{h}$, and $\gamma^{h}$ denote the regularization of the frictionless functional term $\mathcal{K}^{h}\left(\mathbf{u}_{n}^{h}\right)$, the Lagrange multipliers, and a virtual variable, which represent the frictionless contact forces, respectively. The augmented Lagrangian functional $\varrho^{h}$ we use in this paper is given by

$$
\begin{array}{r}
\mathcal{L}^{h}\left(\mathbf{w}^{h}, \gamma^{h}\right) \\
=\int_{\Gamma_{3}}\left(d_{v}^{h}\left(\mathbf{w}^{h}\right) \gamma^{h}+\frac{r}{2}\left|d_{v}^{h}\left(\mathbf{w}^{h}\right)\right|^{2}-\frac{1}{2 r} \operatorname{dist}_{\mathbb{R}^{-}}^{2}\left\{\gamma^{h}+r d_{v}^{h}\left(\mathbf{w}^{h}\right)\right\}\right) d a \\
\forall \mathbf{w}^{h} \in V^{h}, \gamma^{h} \in H_{c}^{h},
\end{array}
$$

where $r$ is a positive penalty coefficient and

$$
\operatorname{dist}_{\mathbb{R}^{-}}(\beta)= \begin{cases}0 & \text { if } \beta>0 \\ -\beta & \text { if } \beta \leq 0\end{cases}
$$

For more details about the Lagrangian method, we refer the reader to $[1,8]$.

The final step consists now into turning Problem 5.4 into an equivalent form, using, respectively, the differentials $D \Phi_{n}^{h}$ and $D \mathcal{L}^{h}$ of the functions $\Phi_{n}^{h}$ and $\mathcal{L}^{h}$. This equivalent form is the following problem.

Problem 5.5. Find $\left\{\mathbf{u}_{n}^{h}\right\}_{n=0, \ldots, N} \subset V^{h}$ and $\left\{\lambda_{n}^{h}\right\}_{n=0, \ldots, N} \subset H_{c}^{h}$ such that $\mathbf{u}_{0}^{h}=$ $p^{h} \mathbf{u}_{0}$ and, for $n=1,2, \ldots, N$,

$$
\begin{array}{r}
\left(D \Phi_{n}^{h}\left(\mathbf{u}_{n}^{h}\right), \mathbf{w}^{h}\right)_{V^{h}}+\left(D \mathcal{L}^{h}\left(\mathbf{u}_{n}^{h}, \lambda_{n}^{h}\right),\left(\mathbf{w}^{h}, \gamma^{h}\right)\right)_{V^{h} \times H_{c}^{h}}=0 \\
\forall \mathbf{w}^{h} \in V^{h}, \gamma^{h} \in H_{c}^{h} .
\end{array}
$$

We here use $(\cdot, \cdot)_{V^{h} \times H_{c}^{h}}$ to denote the canonical inner product on the product Hilbert space $V^{h} \times H_{c}^{h}$. The Lagrangian approach presented above shows that, at each time increment, Problem 5.5 is governed by the system of nonlinear equations 
Frictionless contact of two viscoelastic bodies

$$
\mathbf{A}\left(\mathbf{v}_{n}^{h}\right)+\mathbf{G}\left(\mathbf{u}_{n}^{h}\right)+\mathcal{F}\left(\mathbf{u}_{n}^{h}, \lambda_{n}^{h}\right)=\mathbf{0}
$$

Here the term $\mathbf{A}\left(\mathbf{v}_{n}^{h}\right)+\mathbf{G}\left(\mathbf{u}_{n}^{h}\right)$ represent the gradient of the functional $\Phi_{n}^{h}$ in the direction $\mathbf{u}_{n}^{h}, \mathbf{v}_{n}^{h}$ being given by (5.1), and the term $\mathcal{F}\left(\mathbf{u}_{n}^{h}, \lambda_{n}^{h}\right)$ denotes the gradient of the functional $\mathcal{L}^{h}$ in the direction $\left(\mathbf{u}_{n}^{h}, \lambda_{n}^{h}\right)$. We remark that the volume and surface efforts are contained in the term $\mathbf{G}\left(\mathbf{u}_{n}^{h}\right)$. Moreover, for simplicity, in (5.13) and below we do not indicate the dependence of the operators $\mathbf{A}$ and $\mathbf{G}$ on $h$ and $n$, nor the dependence of the operator $\mathcal{F}$ on $h$.

To solve (5.13), at each time increment, the variables $\left(\mathbf{u}_{n}^{h}, \lambda_{n}^{h}\right)$ are treated simultaneously through a Newton method and therefore in what follows we use $\mathbf{x}_{n}^{h}$ to denote the pair $\left(\mathbf{u}_{n}^{h}, \lambda_{n}^{h}\right)$. Notice that the left-hand side of the system (5.13) contains three terms: the viscous term defined by the operator $\mathbf{A}$, the elastic term given by $\mathbf{G}$, and a nondifferentiable contact term described by $\mathcal{F}$. In the following, to simplify the notation, we will omit the spatial discretization index $h$.

The solution algorithm we use is a combination of the finite-differences and the linear iterations methods. The finite-differences is based on a generalized trapezes $\alpha$-method that we choose here in order to have a better control of the stability of the numerical scheme, while the linear iterations are based on a Newton method. In order to overcome the nondifferentiability involved in the system (5.13), the Newton method has been extended to a generalized Newton method (GNM) (see [2] for details).

The algorithm we have used in the viscoelastic case can be developed in three steps presented as follows.

\section{A prediction step}

This step gives the initial displacement and the velocity by the following formula:

$$
\mathbf{u}_{n+1}^{0}=\mathbf{u}_{n+1}+\mathbf{v}_{n}, \quad \mathbf{v}_{n+1}^{0}=\mathbf{v}_{n}
$$

\section{A Newton linearization step}

At an iteration $i$ of the Newton method, we have

$$
\begin{aligned}
& \mathbf{x}_{n+1}^{i+1}=\mathbf{x}_{n+1}^{i}-\left(\frac{\mathbf{D}_{n+1}^{i}}{\alpha \Delta t}+\mathbf{K}_{n+1}^{i}+\mathbf{T}_{n+1}^{i}\right)^{-1}\left(\mathbf{A}\left(\mathbf{v}_{n+1}^{i}\right)+\mathbf{G}\left(\mathbf{u}_{n+1}^{i}\right)+\mathcal{F}\left(\mathbf{x}_{n+1}^{i}\right)\right), \\
& \mathbf{K}_{n+1}^{i}=D \mathbf{G}\left(\mathbf{u}_{n+1}^{i}\right), \quad \mathbf{D}_{n+1}^{i}=D \mathbf{A}\left(\mathbf{v}_{n+1}^{i}\right), \quad \mathbf{T}_{n+1}^{i} \in \partial \mathcal{F}\left(\mathbf{u}_{n+1}^{i}, \lambda_{n+1}^{i}\right),
\end{aligned}
$$


where $\Delta \mathbf{x}^{i}=\left(\Delta \mathbf{u}^{i}, \Delta \lambda^{i}\right)$ with $\Delta \mathbf{u}^{i}=\mathbf{u}_{n+1}^{i+1}-\mathbf{u}_{n+1}^{i}$ and $\Delta \lambda^{i}=\lambda_{n+1}^{i+1}-\lambda_{n+1}^{i}$. Here $D \mathbf{G}$ and $D \mathbf{A}$ represent the differential of the operators $\mathbf{G}$ and $\mathbf{A}$, respectively, and $\partial \mathcal{F}(\mathbf{x})$ denotes the generalized Jacobian of $\mathcal{F}$ at $\mathbf{x}$. This leads us to solve the resulting linear system

$$
\left(\frac{\mathbf{D}_{n+1}^{i}}{\alpha \Delta t}+\mathbf{K}_{n+1}^{i}+\mathbf{T}_{n+1}^{i}\right) \Delta \mathbf{x}^{i}=-\mathbf{A}\left(\mathbf{v}_{n+1}^{i}\right)-\mathbf{G}\left(\mathbf{u}_{n+1}^{i}\right)-\mp\left(\mathbf{x}_{n+1}^{i}\right) .
$$

We solve the linear system of (5.16) by using a conjugate gradient method with efficient preconditioners to overcome the poor conditioning of the matrix due to the unilateral contact term. For more details, we refer the reader to $[4,27]$.

A correction step

Once the system (5.16) is resolved, we update $\mathbf{x}_{n+1}^{i+1}$ and $\mathbf{v}_{n+1}^{i+1}$ by

$$
\mathbf{x}_{n+1}^{i+1}=\mathbf{x}_{n+1}^{i}+\Delta \mathbf{x}^{i}, \quad \mathbf{v}_{n+1}^{i+1}=\mathbf{v}_{n+1}^{i}+\frac{\Delta \mathbf{u}^{i}}{\alpha \Delta t} .
$$

Notice that similar arguments can be used in order to study the discrete approximation of the elastic contact Problem 4.2. In this case the viscosity term $\mathbf{A}$ vanishes there and we remark that the system (5.13) becomes

$$
\mathbf{G}\left(\mathbf{u}_{n}^{h}\right)+\mathcal{F}\left(\mathbf{u}_{n}^{h}, \lambda_{n}^{h}\right)=\mathbf{0}
$$

We again use a Newton method to solve the system (5.18) with the unknowns $\left(\mathbf{u}_{n}^{h}, \lambda_{n}^{h}\right)$ replaced by $\mathbf{x}_{n}^{h}$ and, again, for simplicity, we omit the index $h$. This leads to a three steps algorithm taking the following form.

\section{A prediction step}

The initial displacement is given by

$$
\mathbf{u}_{n+1}^{0}=\mathbf{u}_{n}
$$

\section{A Newton linearization step}

At an iteration $i$ of the Newton method, we have

$$
\left(\mathbf{K}_{n+1}^{i}+\mathbf{T}_{n+1}^{i}\right) \Delta \mathbf{x}^{i}=-\mathbf{G}\left(\mathbf{u}_{n+1}^{i}\right)-\mathcal{F}\left(\mathbf{x}_{n+1}^{i}\right) .
$$


A correction step

We now update $\mathbf{x}_{n+1}^{i+1}$ :

$$
\mathbf{x}_{n+1}^{i+1}=\mathbf{x}_{n+1}^{i}+\Delta \mathbf{x}^{i} .
$$

To conclude, to solve the elastic problem, we use the same algorithm as in the viscoelastic case in which we just take the viscosity operator $\mathbf{A}$ as zero.

\section{Numerical results}

In this section, we illustrate our theoretical results by numerical simulations in the study of two-dimensional test problems. In both examples, the viscoelastic bodies are supposed to occupy polygonal domains in the reference configuration and the potential contact surfaces are straight lines. Based on the numerical simulations presented in this section, we strongly believe that the Signorini frictionless condition matches with this particular geometries and it represents a good approximation of the contact process, at least for contact processes which occur in a short interval of time.

In both examples, we consider Problem 2.1 in the case when the body forces vanish, that is, $\boldsymbol{\varphi}_{1}^{m}=\mathbf{0}$ for $m=1,2$. We use a discretization by linear piecewise functions for the space $V^{h}$ and a uniform partition of the time interval. We compute the numerical solution both in the viscoelastic and in the elastic cases in order to illustrate the convergence result in Theorem 4.3. Moreover, we consider linear elastic and linear viscoelastic materials. The elasticity tensor $\mathcal{G}^{m}$ and the viscosity tensor $\mathcal{A}^{m}$ are given by

$$
\begin{gathered}
\left(\mathcal{G}^{m} \boldsymbol{\tau}\right)_{\alpha \beta}=\frac{E}{1-v^{2}}\left(\tau_{11}+\tau_{22}\right) \delta_{\alpha \beta}+\frac{E}{1+v} \tau_{\alpha \beta}, \quad 1 \leq \alpha, \beta \leq 2, \\
\left(\mathcal{A}^{m} \boldsymbol{\tau}\right)_{\alpha \beta}=\mu\left(\tau_{11}+\tau_{22}\right) \delta_{\alpha \beta}+\eta \tau_{\alpha \beta}, \quad 1 \leq \alpha, \beta \leq 2,
\end{gathered}
$$

where $E$ is the Young modulus, $v$ is the Poisson ratio of the material, $\mu, \eta$ are viscosity constants, and $m=1,2$. It is straightforward to see that such kind of tensors satisfy conditions (4.1) and (2.12), respectively.

To visualize the stresses, we use the Tresca criteria which is given in the case of plane stresses by the formula

$$
|\boldsymbol{\sigma}|_{\operatorname{Tr}}=\frac{1}{2} \max _{i, j, i \neq j}\left|\sigma_{i}-\sigma_{j}\right|,
$$




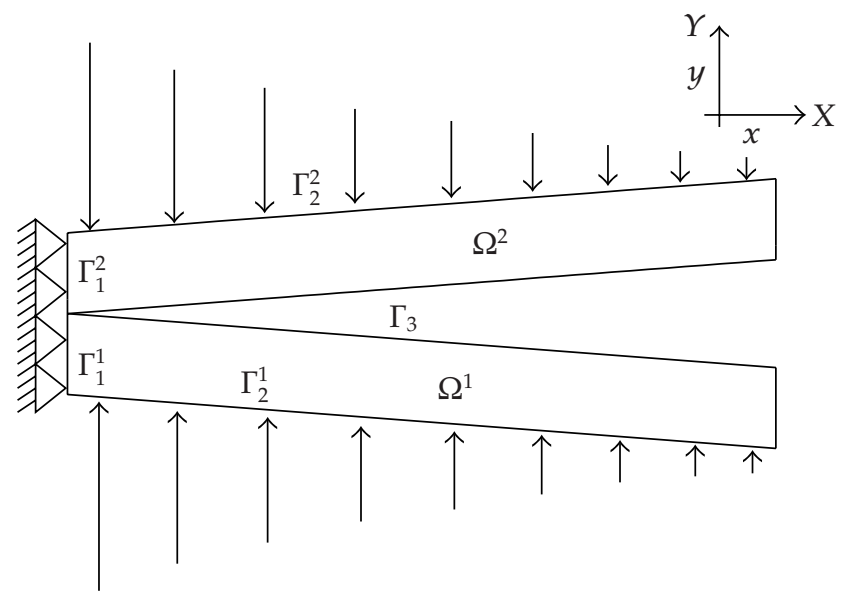

FIGURE 6.1. Initial configuration in the first two-dimensional example.

where $\sigma_{i}, i=1,2$ are the principal directions associated to a stress field $\sigma \in \mathbb{S}^{2}$.

For computation in the two examples below, we have used the following data: $T=10 \mathrm{~s}, \boldsymbol{\varphi}_{1}^{m}=0 \mathrm{~N} / \mathrm{m}^{2}, E=1 \mathrm{~N} / \mathrm{m}^{2}, v=0.3, \mathbf{u}_{0}=0 \mathrm{~m}$. The surface tractions and the viscosity coefficients will be specified later.

\subsection{First two-dimensional example}

We consider the physical setting presented in Figure 6.1. Here, the two bodies are assumed to be in an oblique position. Notice that in this case, there exists a nonzero gap between the contact surfaces; however, our results above can be extended in this case too. The bodies are clamped on their respective parts $\Gamma_{1}^{1}=\{0\} \times(1.5,3)$ and $\Gamma_{1}^{2}=\{0\} \times(3,4.5)$. The intensity of the surface tractions depends only on the first component of the point where they are applied and decreases in the X-direction. Such intensity is given by the formula: $-0.00004(10-x)^{2}$, where $x$ symbolizes the first component of the spatial point.

We made computations both for the viscoelastic and elastic case. For the viscoelastic cases, we successively choose the following viscosity coefficients: $(\mu, \eta)=(1,0.6),(\mu, \eta)=(0.5,0.3),(\mu, \eta)=(0.25,0.15)$, and $(\mu, \eta)=$ $(0.15,0.075)$. Here and below, for simplicity, we do not indicate the units of the constants $\mu$ and $\eta$. The deformed configuration of the two bodies at final time are plotted in Figures 6.2, 6.3, 6.4, 6.5, and 6.6.

The Tresca criteria $|\sigma|_{T r}$ at the final time for the viscoelastic case $(\mu$, $\eta)=(0.5,0.3)$ and for the elastic case are presented in Figure 6.7, on the left-hand side and right-hand side respectively. Here, the clear nuances 
596 Frictionless contact of two viscoelastic bodies

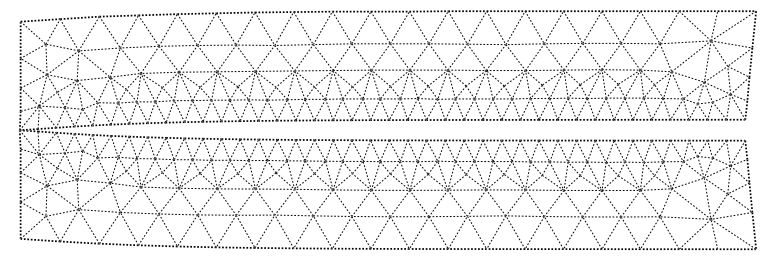

FIGURE 6.2. Deformed configuration of the two viscoelastic bodies for $\mu=1.0 \mathrm{~ns} / \mathrm{m}^{2}$ and $\eta=0.6 \mathrm{~ns} / \mathrm{m}^{2}$.



FIGURE 6.3. Deformed configuration of the two viscoelastic bodies for $\mu=0.5 \mathrm{~ns} / \mathrm{m}^{2}$ and $\eta=0.3 \mathrm{~ns} / \mathrm{m}^{2}$.

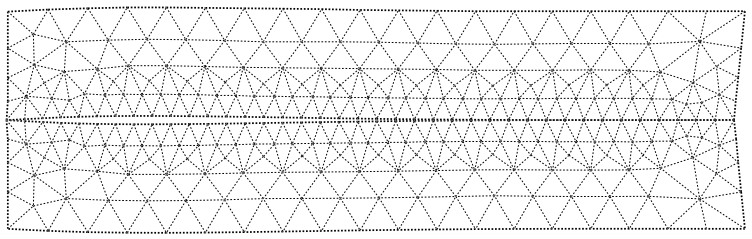

FIGURE 6.4. Deformed configuration of the two viscoelastic bodies for $\mu=0.25 \mathrm{~ns} / \mathrm{m}^{2}$ and $\eta=0.15 \mathrm{~ns} / \mathrm{m}^{2}$.

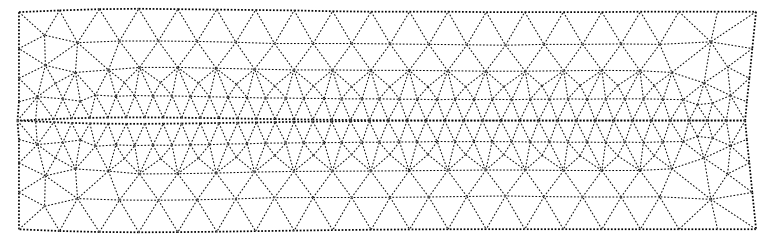

FIGURE 6.5. Deformed configuration of the two viscoelastic bodies for $\mu=0.15 \mathrm{~ns} / \mathrm{m}^{2}$ and $\eta=0.075 \mathrm{~ns} / \mathrm{m}^{2}$.

of gray represent the region where the stresses are more important and the dark gray represents the region where the stresses are less important.

It follows from these numerical simulations that the viscosity plays an important role since it attenuates the efforts due to the forces. This example illustrates also the fact that the elastic problem may be considered as a limit case of the viscoelastic one, as proved in Theorem 4.3. 


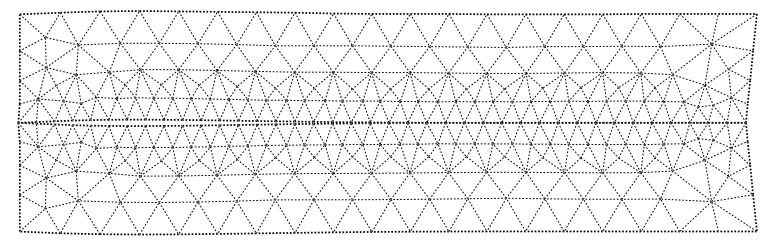

FIGURE 6.6. Deformed configuration of the two elastic bodies.
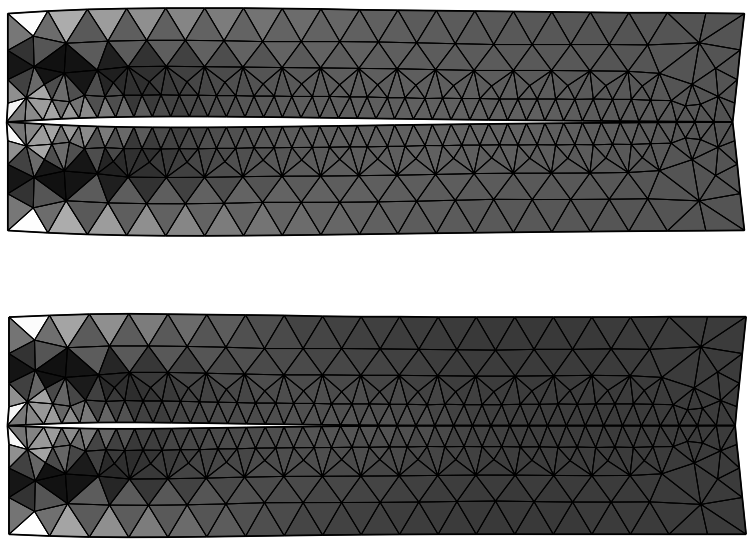

FIGURE 6.7. Tresca criteria for the stresses in the viscoelastic $(0.5,0.3)$ and elastic cases.

\subsection{Second two-dimensional example}

For the second example, the physical setting is shown in Figure 6.8. Here the bodies are supposed to be imbricated in their reference configuration. The first body $\Omega^{1}$ is assumed to be clamped on $\Gamma_{1}^{1}=\{0\} \times(0,2) \cup\{10\} \times$ $(0,2)$ of its boundary while the second body $\Omega^{2}$ is clamped on $\Gamma_{1}^{2}=\{0\} \times$ $(2,3) \cup\{10\} \times(2,3)$ of its boundary. No surface forces are acting on the part $\Gamma_{2}^{2}=(0,10) \times\{3\}$ and a constant force of intensity $2.05 \times 10^{-3} \mathrm{~ns} / \mathrm{m}^{2}$ is acting on $\Gamma_{2}^{1}=(0,10) \times\{0\}$ in the negative sense of the $Y$-axis. The common contact surface $\Gamma_{3}$ is highlighted in bold on Figure 6.8.

As in the previous example, we performed simulations both in the viscoelastic and elastic cases. For the viscoelastic case, we successively used our algorithm with the viscosity coefficients $(\mu, \eta)=(1.0,0.4),(\mu, \eta)=$ $(0.5,0.2),(\mu, \eta)=(0.25,0.1)$, and $(\mu, \eta)=(0.125,0.05)$. The results at the end of the simulation are illustrated in Figures 6.9, 6.10, 6.11, 6.12, and 6.13 , which represent the deformed configuration of the two bodies at the final time. 


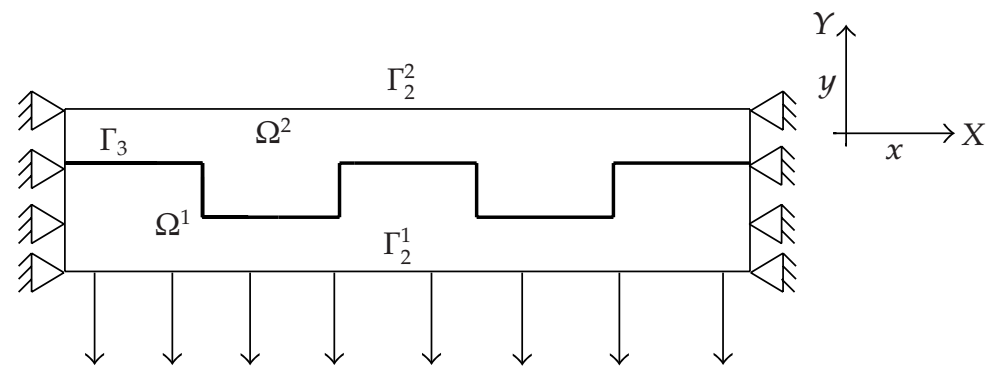

FIGURE 6.8. Initial configuration in the second two-dimensional example.

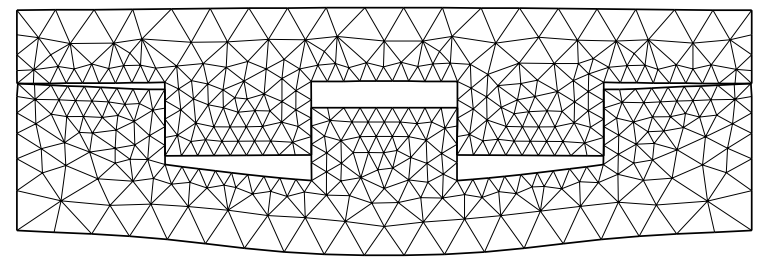

FIGURE 6.9. Deformed configuration of the two viscoelastic bodies for $\mu=1.0 \mathrm{~ns} / \mathrm{m}^{2}$ and $\eta=0.4 \mathrm{~ns} / \mathrm{m}^{2}$.

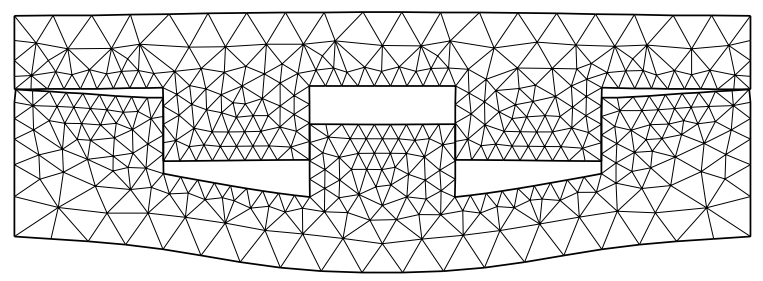

FIGURE 6.10. Deformed configuration of the two viscoelastic bodies for $\mu=0.5 \mathrm{~ns} / \mathrm{m}^{2}$ and $\eta=0.2 \mathrm{~ns} / \mathrm{m}^{2}$.

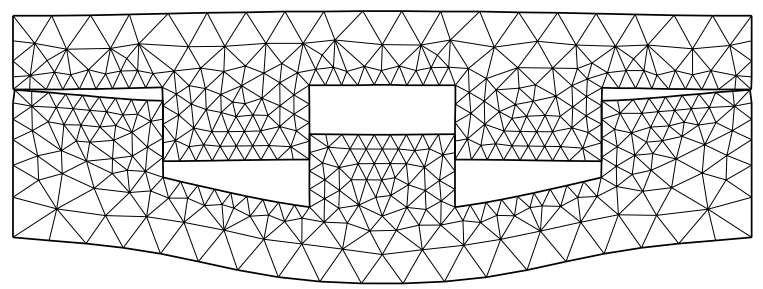

FIGURE 6.11. Deformed configuration of the two viscoelastic bodies for $\mu=0.25 \mathrm{~ns} / \mathrm{m}^{2}$ and $\eta=0.1 \mathrm{~ns} / \mathrm{m}^{2}$. 


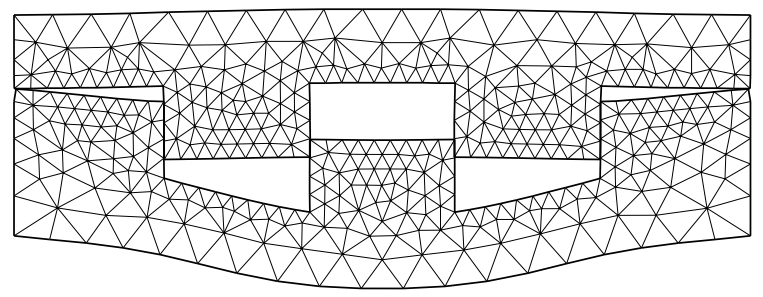

FIGURE 6.12. Deformed configuration of the two viscoelastic bodies for $\mu=0.125 \mathrm{~ns} / \mathrm{m}^{2}$ and $\eta=0.05 \mathrm{~ns} / \mathrm{m}^{2}$.

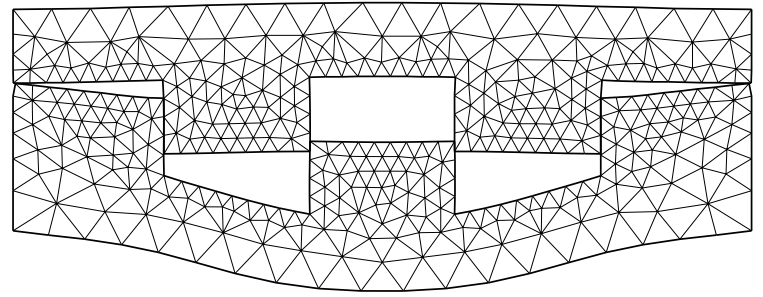

FIGURE 6.13. Deformed configuration of the two elastic bodies.
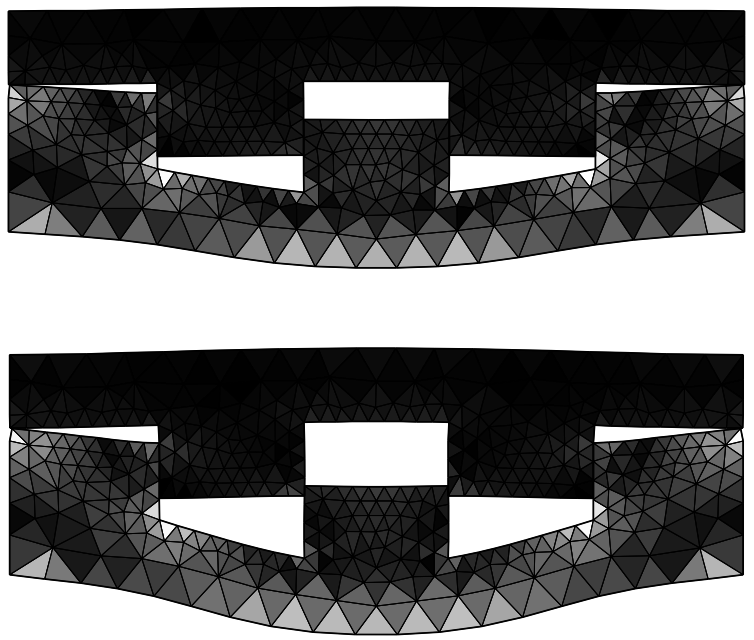

FIgURE 6.14. Tresca criteria for the stresses in the viscoelastic $(0.5,0.2)$ and elastic cases. 
The Tresca criteria $|\boldsymbol{\sigma}|_{T r}$ at the final time $T$ for the viscoelastic case $(\mu, \eta)=(0.5,0.2)$ and for the elastic case are presented in Figure 6.14, on the left-hand side and right-hand side, respectively. Again, the clear nuances of gray represent the region where the stresses are more important and the dark gray represents the region where the stresses are less important.

We notice that the numerical simulations presented in Figures 6.9, $6.10,6.11,6.12$, and 6.13 are in agreement with the theoretical result of Theorem 4.3 since they show that the elastic case is a limit of the viscoelastic case as the viscosity coefficients converge to zero.

\section{Conclusions}

We presented a model for the quasistatic process of frictionless contact between two viscoelastic bodies within the linear theory of small displacements. The variational inequality for the contact problem was derived, and then it was coupled with the constitutive law and the initial condition. For this mathematical problem, we established the existence of the unique weak solution and we studied its behavior, as the viscosity tensor converges to zero. Then, we presented a fully discrete scheme for the numerical approximations of the problem as a basis for a computer code. Two examples were computed using this code. The computer code was found to behave well, and the numerical solutions seem accurate and interesting. We remark that three problems, which are outside of the aim of this paper, are left open.

The first one concerns the modeling and more precisely the description of the evolutionary contact condition between two deformable bodies. Clearly, the classical Signorini frictionless condition we used here is quite restrictive since it does not provide an accurate description of the tangential motion, and therefore it may be of interest to consider more realistic contact models in the future. However, currently, very few results on this topic are available. Also, models vary from author to author and from paper to paper, and there is no doubt that a closer look at the physics of contacting surfaces is needed.

The second open problem concerns a regularity result. Indeed, in Theorem 3.2, we obtained the basic regularity of the solution, $\mathbf{u}_{\theta} \in$ $W^{1, \infty}(0, T ; V)$, and we used it in the first two convergence results presented in Theorem 4.3. However, to obtain the last convergence result in the above theorem, we need an additional regularity of the solution, $\mathbf{u}_{\theta} \in$ $W^{2,2}(0, T ; V)$, that we assumed as given. Deriving this regularity from appropriate regularity assumption imposed on the input data should be of real interest since the field of regularity of solutions in contact mechanics contains very few results, is wide open, and its progress is likely to be slow. 
The third open problem concerns the numerical algorithm we used. Although recent progress in the study of convergence and error estimates for the fully discrete scheme used in contact mechanics is impressive (see, e.g., the list of references in [15]), many open problems remain and, to the best of our knowledge, there exist no theoretical results concerning the convergence of the fully discrete scheme associated with the augmented Lagrangian approach we used in this paper. However, the results in the literature strongly suggest that this method converges and it is very accurate and reliable.

We conclude that the results presented in this paper represent a step in the study of quasistatic contact problems between two deformable bodies, which inherently are nonlinear, diverse, and rather complex, and give rise to new and interesting mathematical models which need to be solved in the future.

\section{References}

[1] P. Alart, Méthode de Newton généralisée en mécanique du contact, J. Math. Pures Appl. (9) 76 (1997), no. 1, 83-108.

[2] P. Alart and A. Curnier, A mixed formulation for frictional contact problems prone to Newton like solution methods, Comput. Methods Appl. Mech. Engrg. 92 (1991), no. 3, 353-375.

[3] L.-E. Andersson, Existence results for quasistatic contact problems with Coulomb friction, Appl. Math. Optim. 42 (2000), no. 2, 169-202.

[4] M. Barboteu, Contact, frottement et techniques de calcul parallèle, Ph.D. thesis, University of Montpellier II, Montpellier, 1999.

[5] M. Barboteu, W. Han, and M. Sofonea, A frictionless contact problem for viscoelastic materials, J. Appl. Math. 2 (2002), no. 1, 1-21.

[6] V. Barbu, Optimal Control of Variational Inequalities, Research Notes in Mathematics, vol. 100, Pitman, Massachusetts, 1984.

[7] J. Chen, W. Han, and M. Sofonea, Numerical analysis of a contact problem in ratetype viscoplasticity, Numer. Funct. Anal. Optim. 22 (2001), no. 5-6, 505527.

[8] A. Curnier, Q. C. He, and A. Klarbring, Continuum mechanics modelling of large deformation contact with friction, Contact Mechanics (M. Raous, M. Jean, and J. J. Moreau, eds.), Plenum Press, New York, 1995, pp. 145-158.

[9] G. Duvaut and J.-L. Lions, Inequalities in Mechanics and Physics, SpringerVerlag, Berlin, 1976.

[10] J. R. Fernández García, W. Han, M. Shillor, and M. Sofonea, Numerical analysis and simulations of quasistatic frictionless contact problems, Int. J. Appl. Math. Comput. Sci. 11 (2001), no. 1, 205-222.

[11] G. Fichera, Problemi elastostatici con vincoli unilaterali: Il problema di Signorini con ambigue condizioni al contorno, Atti Accad. Naz. Lincei Mem. Cl. Sci. Fis. Mat. Natur. Sez. I (8) 7 (1963/1964), 91-140.

[12] _ Boundary value problems of elasticity with unilateral constraints, Encyclopedia of Physics, vol. VI a/2, Springer, Berlin, 1972. 


\section{Frictionless contact of two viscoelastic bodies}

[13] I. G. Goryacheva, Contact Mechanics in Tribology, Solid Mechanics and Its Applications, vol. 61, Kluwer Academic Publishers, Dordrecht, 1998.

[14] W. Han and M. Sofonea, Numerical analysis of a frictionless contact problem for elastic-viscoplastic materials, Comput. Methods Appl. Mech. Engrg. 190 (2000), no. 1-2, 179-191.

[15] _ Quasistatic Contact Problems in Viscoelasticity and Viscoplasticity, AMS/IP Studies in Advanced Mathematics, vol. 30, American Mathematical Society, Rhode Island, 2002.

[16] J. Haslinger and I. Hlaváček, Contact between elastic bodies. I. Continuous problems, Apl. Mat. 25 (1980), no. 5, 324-347.

[17] _ Contact between elastic bodies. II. Finite element analysis, Apl. Mat. 26 (1981), no. 4, 263-290.

[18]__ Contact between elastic bodies. III. Dual finite element analysis, Apl. Mat. 26 (1981), no. 5, 321-344.

[19] I. Hlaváček, J. Haslinger, J. Nečas, and J. Lovíšek, Solution of Variational Inequalities in Mechanics, Applied Mathematical Sciences, vol. 66, SpringerVerlag, New York, 1988.

[20] J. Jarušek, Dynamical contact problems for bodies with a singular memory, Boll. Un. Mat. Ital. A (7) 9 (1995), no. 3, 581-592.

[21] _ Dynamic contact problems with given friction for viscoelastic bodies, Czechoslovak Math. J. 46(121) (1996), no. 3, 475-487.

[22] _ Remark to dynamic contact problems for bodies with a singular memory, Comment. Math. Univ. Carolin. 39 (1998), no. 3, 545-550.

[23] J. Jarušek and C. Eck, Dynamic contact problems with small Coulomb friction for viscoelastic bodies. Existence of solutions, Math. Models Methods Appl. Sci. 9 (1999), no. 1, 11-34.

[24] N. Kikuchi and J. T. Oden, Contact Problems in Elasticity: A Study of Variational Inequalities and Finite Element Methods, SIAM Studies in Applied Mathematics, vol. 8, SIAM, Pennsylvania, 1988.

[25] J. A. C. Martins and M. D. P. M. Marques (eds.), Contact Mechanics, Solid Mechanics and Its Applications, vol. 103, Kluwer Academic Publishers Group, Dordrecht, 2002.

[26] J. Nečas and I. Hlaváček, Mathematical Theory of Elastic and Elasto-Plastic Bodies: An Introduction, Studies in Applied Mechanics, vol. 3, Elsevier Scientific Publishing, Amsterdam, 1980.

[27] G. Pietrzak and A. Curnier, Large deformation frictional contact mechanics: continuum formulation and augmented Lagrangian treatment, Comput. Methods Appl. Mech. Engrg. 177 (1999), no. 3-4, 351-381.

[28] M. Raous, M. Jean, and J. J. Moreau (eds.), Contact Mechanics, Plenum Press, New York, 1995.

[29] M. Rochdi and M. Sofonea, On frictionless contact between two elasticviscoplastic bodies, Quart. J. Mech. Appl. Math. 50 (1997), no. 3, 481-496.

[30] M. Shillor (ed.), Special issue on recent advances in contact mechanics, Math. Comput. Modelling 28 (1998), no. 4-8.

[31] M. Shillor, M. Sofonea, and J. J. Telega, Models and Analysis of Quasistatic Contact, in press.

[32] A. Signorini, Sopra alcune questioni di elastostatica, Atti della Società Italiana per il Progresso delle Scienze, 1933. 
[33] M. Sofonea, On a contact problem for elastic-viscoplastic bodies, Nonlinear Anal. 29 (1997), no. 9, 1037-1050.

[34] J. J. Telega, Variational inequalities in contact problems of mechanics, Contact Mechanics of Surfaces (Z. Mróz, ed.), Ossolineum, Wroclaw, 1988, pp. 51165.

[35] P. Wriggers and P. Panagiotopoulos (eds.), New Developments in Contact Problems, Springer, Wien, 1999.

M. Barboteu: Laboratoire de Théorie des Systèmes, Université de Perpignan, 52 avenue de Villeneuve, 66860 Perpignan, France

T.-V. Hoarau-Mantel: Laboratoire de Théorie des Systèmes, Université de Perpignan, 52 avenue de Villeneuve, 66860 Perpignan, France

M. Sofonea: Laboratoire de Théorie des Systèmes, Université de Perpignan, 52 avenue de Villeneuve, 66860 Perpignan, France 


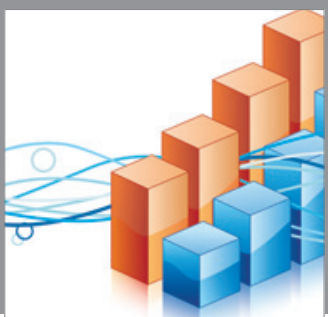

Advances in

Operations Research

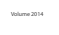

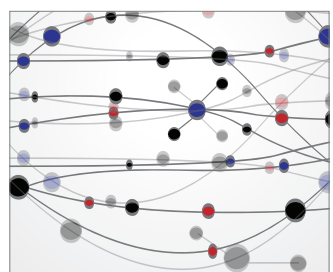

\section{The Scientific} World Journal
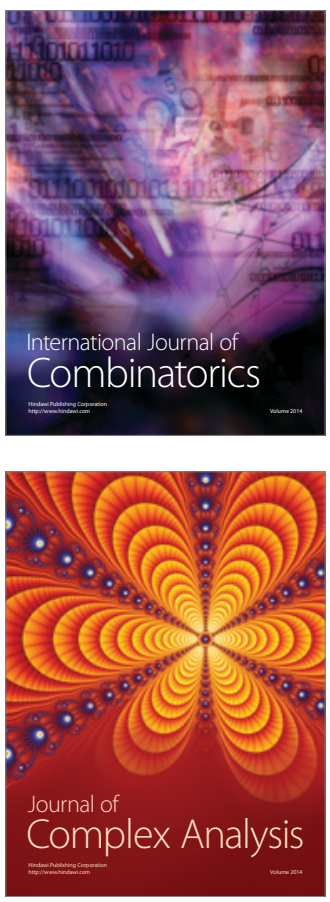

International Journal of

Mathematics and

Mathematical

Sciences
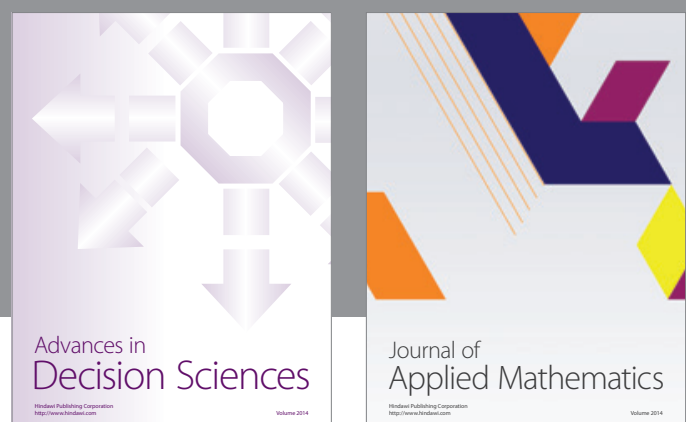

Journal of

Applied Mathematics
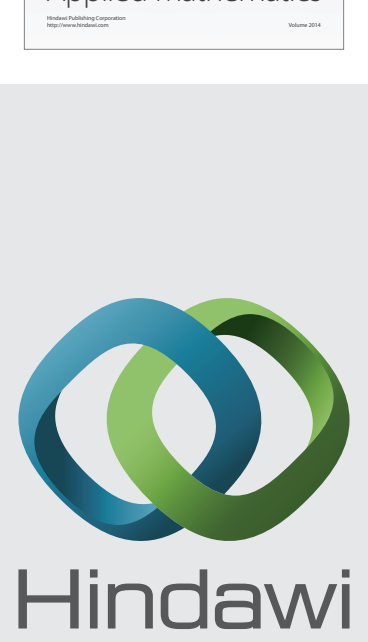

Submit your manuscripts at http://www.hindawi.com
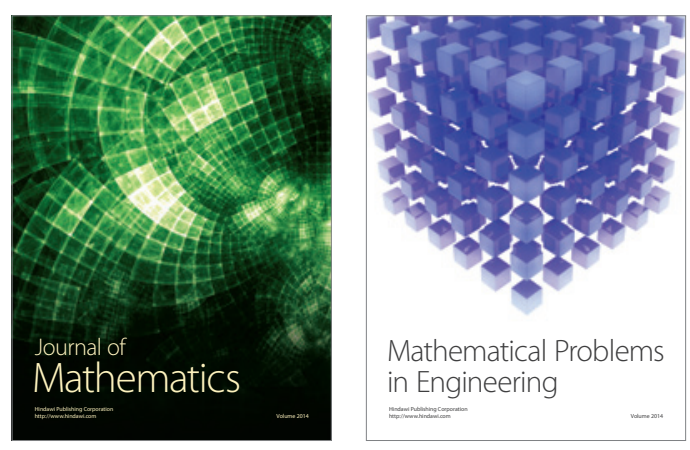

Mathematical Problems in Engineering
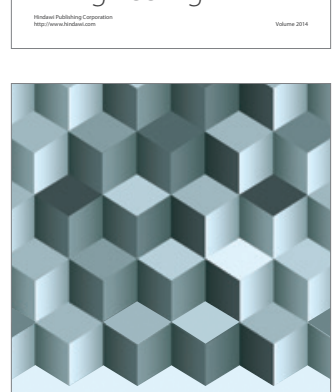

Journal of

Function Spaces
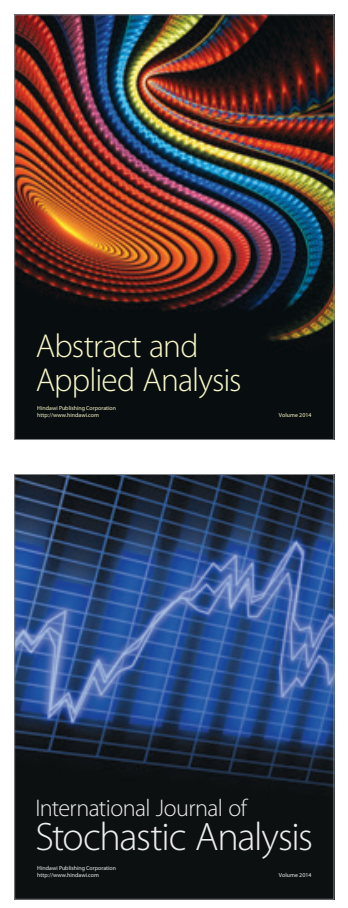

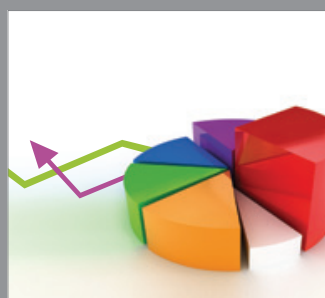

ournal of

Probability and Statistics

Promensencen
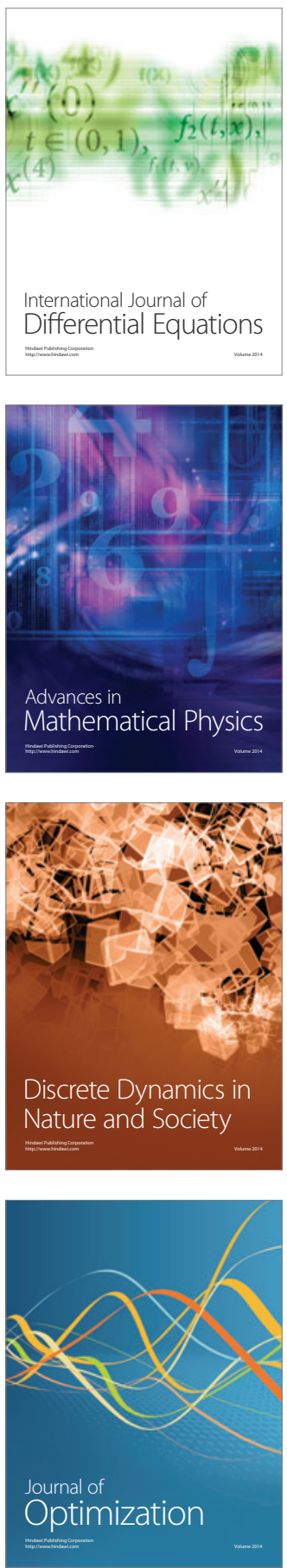